\title{
THE SOLAR WIND AND SUPRATHERMAL ION COMPOSITION INVESTIGATION ON THE WIND SPACECRAFT
}

\author{
G. GLOECKLER \\ Department of Physics, University of Maryland, College Park, MD, U.S.A. \\ H. BALSIGER, A. BÜRGI, P. BOCHSLER \\ Physikalisches Institut, Universität Bern, Bern, Switzerland
}

\section{A. FISK}

Department of Atmospheric, Oceanic and Space Sciences, University of Michigan, Ann Arbor, MI, U.S.A.

\section{A. B. GALVIN}

Department of Physics, University of Maryland, College Park, MD, U.S.A.

\section{J. GEISS}

Physikalisches Institut, Universität Bern, Bern, Switzerland

\section{F. GLIEM}

Institut für Datenverarbeitungsanlagen, Technische Universität Braunschweig, Braunschweig,

Germany

D. C. HAMILTON

Department of Physics, University of Maryland, College Park, MD, U.S.A.

T. E. HOLZER

High Altitude Observatory (NCAR), Boulder, CO, U.S.A.

\section{HOVESTADT}

Max-Planck-Institut für Extraterrestrische Physik, Garching, Germany

F. M. IPAVICH

Department of Physics, University of Maryland, College Park, MD, U.S.A.

E. KIRSCH

Max-Planck-Institut für Aeronomie, Katlenburg-Lindau, Germany

R. A. LUNDGREN

Department of Physics, University of Maryland, College Park, MD, U.S.A.

K. W. OGILVIE

National Aeronautics and Space Administration/Goddard Space Flight Center, Greenbelt, MD, U.S.A.

R. B. SHELDON

Department of Physics, University of Maryland, College Park, MD, U.S.A.; and Physikalisches Institut, Universität Bern, Bern, Switzerland

and

B. WILKEN

Max-Planck-Institut für Aeronomie, Katlenburg-Lindau, Germany

(Received 26 March, 1993) 


\begin{abstract}
The Solar Wind and Suprathermal Ion Composition Experiment (SMS) on WIND is designed to determine uniquely the elemental, isotopic, and ionic-charge composition of the solar wind, the temperatures and mean speeds of all major solar-wind ions, from $\mathrm{H}$ through $\mathrm{Fe}$, at solar wind speeds ranging from $175 \mathrm{kms}^{-1}$ (protons) to $1280 \mathrm{kms}^{-1}\left(\mathrm{Fe}^{+8}\right)$, and the composition, charge states as well as the 3-dimensional distribution functions of suprathermal ions, including interstellar pick-up $\mathrm{He}^{+}$, of energies up to $230 \mathrm{keV} / \mathrm{e}$. The experiment consists of three instruments with a common Data Processing Unit. Each of the three instruments uses electrostatic analysis followed by a time-of-flight and, as required, an energy measurement. The observations made by SMS will make valuable contributions to the ISTP objectives by providing information regarding the composition and energy distribution of matter entering the magnetosphere. In addition SMS results will have an impact on many areas of solar and heliospheric physics, in particular providing important and unique information on: (i) conditions and processes in the region of the corona where the solar wind is accelerated; (ii) the location of the source regions of the solar wind in the corona; (iii) coronal heating processes; (iv) the extent and causes of variations in the composition of the solar atmosphere; (v) plasma processes in the solar wind; (vi) the acceleration of particles in the solar wind; and (vii) the physics of the pick-up process of interstellar He as well as lunar particles in the solar wind, and the isotopic composition of interstellar helium.
\end{abstract}

\title{
1. Introduction
}

The Solar Wind and Suprathermal Ion Composition (SMS) Experiment will make major contributions to the global objectives of ISTP. It is designed to measure, under all conceivable solar wind flow conditions, (1) the elemental and isotopic composition of solar wind ions, and (2) the differential energy spectra, abundances and ionization or charge states of all dominant ion species from $\mathrm{H}$ to $\mathrm{Fe}$ with energies between 0.5 and $230 \mathrm{keV} / \mathrm{e}$. From the differential energy spectra we will derive routinely the bulk speeds, densities and kinetic temperatures of the dominant solar wind ion species, and the charge state measurements will allow us to infer coronal temperatures and to identify plasma sources of supra-thermal ion populations. This experiment will also provide in-ecliptic, one AU baseline measurements of solar wind composition and conditions as well as the density of pick-up helium (and probably oxygen and neon), important for interpretation of results from solar wind instruments on the Ulysses solar polar mission, as well as from space and ground based remote sensing observations of the Sun. To achieve these broad measurement objectives and to cover the huge $\left(10^{15}\right)$ intensity dynamic range requires three separate sensors: the Solar Wind Ion Composition Spectrometer (SWICS), the high-resolution MASS spectrometer (MASS), and the Supra-Thermal Ion Composition Spectrometer (STICS), serviced by a common Data Processing Unit (DPU).

With SWICS we will determine the abundance of about forty different ions in the solar wind. We will be able to characterize mass distribution and MHD flow conditions upstream of the Earth by measuring with SWICS the differential energy spectra of $\mathrm{H}^{+}$and $\mathrm{He}^{++}$every few minutes and less frequently for the rarer ions (C, $\mathrm{O}, \mathrm{Ne}, \mathrm{Mg}, \mathrm{Si}$, and $\mathrm{Fe}$ ). The SWICS spectrometer makes use of energy/charge analysis and post-acceleration up to $30 \mathrm{keV} / \mathrm{e}$, followed by a time-of-flight vs 
energy measurement to determine both the mass/charge and mass of ions from 0.5 to $30 \mathrm{keV} / \mathrm{e}$. Post-acceleration is required in order to measure the mass of ions of solar wind energies, which otherwise would fall below the threshold of the solid-state detector.

Using STICS we will characterize the interplanetary supra-thermal ion populations by measuring the three-dimensional distribution functions of $\mathrm{H}, \mathrm{He}, \mathrm{C}, \mathrm{O}$, $\mathrm{Si}$, and $\mathrm{Fe}$ for direct comparison with ion populations inside the magnetosphere. Furthermore, STICS will allow us to examine interstellar and lunar pick-up ions in considerable detail and to map the longitudinal distribution of interstellar neutral helium at $1 \mathrm{AU}$. STICS uses energy/charge analysis followed by the time-of-flight vs energy technique to determine both the mass/charge and mass of ions from 30 to $230 \mathrm{keV} / \mathrm{e}$, and mass $/$ charge down to $\sim 8 \mathrm{keV} / \mathrm{e}$. STICS has a large geometrical factor (required to detect the low fluxes of supra-thermal ions). STICS also has a nearly $4 \pi$ viewing geometry affording the capability of measuring three-dimensional distribution function of supra-thermal ions, including high-velocity tails of solar wind ion distributions, diffuse ions, shock-accelerated ions and pick-up ions.

MASS will be the first instrument flown capable of high-mass resolution $(M / \Delta M>100)$ necessary for measurements of the solar wind elemental and isotopic composition. MASS will characterize in detail the matter entering the magnetosphere by measuring the elemental and isotopic composition of the solar wind. MASS uses energy/charge analysis and acceleration/deceleration, followed by a time-of-flight measurement in a retarding, quadratically changing electric potential.

The design, fabrication and testing of the SMS experiment and its ground support equipment was a collaborative effort involving the University of Maryland (STICS sensor and STICS analog electronics, SWICS TOF telescope, high voltage system and interface boards, MASS instrument TOF system, high voltage system, electronics, power supplies and structure, GSE software, and overall responsibility for the SMS experiment), the University of Bern (SWICS deflection analyzer and external support structure, MASS deflection analyzer, and accelerator calibration support), the Max-Planck-Institut fur Aeronomie (SWICS analog electronics and low voltage converter), and the Technical University of Braunschweig (DPU and instrument GSE's).

\section{Major Scientific Objectives}

In addition to the supporting the overall GGS/ISTP objectives by providing the instantaneous characteristics of matter entering the Earth's magnetosphere through measurements of the mass, charge state and energy distributions of solar wind and supra-thermal ions, the unique features of the WIND mission, including its lunar swing-by orbit and its complement of science instruments, make it possible to address a number of science topics and perform correlative studies with other 
WIND investigations. These include the study of dynamic processes in the fore shock region producing reflected, diffuse, and upstream ions, acceleration of e.g. pick-up $\mathrm{He}^{+}$, and wave-particle interactions of heavy ions. Some of the other major scientific problems the SMS investigation will address are listed below.

(1) Determine solar abundances by measuring with MASS the elemental and isotopic composition of the solar wind.

(2) Study solar wind acceleration, especially of minor ions using solar wind composition and charge state measurements made with SWICS.

(3) Study physical processes in the solar atmosphere, such as atom-ion separation in the upper chromosphere, by measuring with MASS the abundances of all elements below $\mathrm{Ni}$, thus spanning the full range of first ionization potentials.

(4) Characterize the physical properties of the acceleration regions (in the lower corona), by measuring with SWICS the solar wind charge state distributions of several ion species (e.g. C, O, Mg, $\mathrm{Si}$, and $\mathrm{Fe}$ ).

(5) Study plasma processes affecting the solar wind kinetic properties and producing supra-thermal ions by measuring with both SWICS and STICS the energy spectra and distribution functions of major ion species from $\sim 0.5$ to $\sim 230 \mathrm{keV} / \mathrm{e}$.

(6) Study interplanetary acceleration mechanisms producing shock-accelerated energetic storm particle events and upstream ions using measurements made with STICS of the three- dimensional distribution functions of $\mathrm{H}, \mathrm{He}, \mathrm{C}, \mathrm{O}, \mathrm{Si}$, and $\mathrm{Fe}$, and of corresponding species in the solar wind with SWICS.

(7) Study the pick-up process of interstellar and lunar neutrals and the acceleration of pick-up ions by interplanetary shocks and the bow shock by measuring with STICS the three- dimensional distribution functions of, e.g., interstellar $\mathrm{He}^{+}$ and lunar $\mathrm{Al}^{+}$.

The major scientific objectives of the SMS investigation are discussed in some detail below.

\subsection{STUDIES OF GEOSPACE}

\subsubsection{Origin of Magnetospheric Plasma}

The solar wind and the terrestrial ionosphere are the principal sources of hot plasma in the magnetosphere. There has been accumulating, but as yet not definitive, evidence that the two sources contribute roughly comparable numbers of ions to the magnetosphere but that depending on geomagnetic activity and phase of the solar cycle, either source may dominate at a particular time (Balsiger et al., 1980; Lennartsson and Shelley, 1986; Chappel et al., 1987; Gloeckler and Hamilton, 1987). The two sources are distinguished by their ionic charge composition. Particles of ionospheric origin should consist mainly of $\mathrm{H}^{+}, \mathrm{O}^{+},{ }^{4} \mathrm{He}^{+}$, and $\mathrm{O}^{+2}$, in varying proportion, whereas solar wind ions are mainly $\mathrm{H}^{+},{ }^{4} \mathrm{He}^{+2}, \mathrm{C}^{+6}$, and $\mathrm{O}^{+6}$. 
The difficulties in using ion composition measurements to determine the origin of the plasma in a particular region of the magnetosphere and at a particular time should not be minimized. There are many complex and competing processes occurring. The composition of the magnetospheric particles depends on the composition and strengths of the sources, on ion fractionation during entry, acceleration, and transport; and on differences in loss rates for different ion species (e.g., by charge exchange or resonant wave-particle interactions).

One of the difficulties in evaluating the relative importance of the solar wind and ionospheric sources is that both are strong sources of $\mathrm{H}^{+}$, which typically is the most abundant species in the magnetosphere. $\mathrm{He}^{+2}$ would be a reliable tracer of the solar wind and $\mathrm{He}^{+}$of the ionosphere except that charge exchange with the geocorona can become important depending on the location and residence time of the ions in the magnetosphere with the result that, below a few hundred $\mathrm{keV}, \mathrm{He}^{+2}$ is preferentially converted to $\mathrm{He}^{+}$.

It is essential, then, for unambiguous tracing of the origin of the plasma in different regions of the magnetosphere to measure the composition of not only the most abundant ions, $\mathrm{H}^{+}, \mathrm{He}^{+2}$, and $\mathrm{O}^{+}$, but also minor constituents such as the solar wind ions $\mathrm{C}^{+6}$ and $\mathrm{O}^{+6}$. Indeed, the most sensitive test of a solar wind or an ionospheric origin could be the ${ }^{4} \mathrm{He} /{ }^{\beta} \mathrm{He}$ isotopic ratio in the plasma. In the solar wind ${ }^{4} \mathrm{He} /{ }^{3} \mathrm{He} \sim 2500$, whereas for ionospheric particles, ${ }^{4} \mathrm{He} /{ }^{3} \mathrm{He} \sim 10^{6}$.

In the solar wind, the SWICS and MASS sensors can make the required ionic and isotopic composition measurements as a function of time, since the solar wind composition varies with time. Simultaneous measurements made within the magnetosphere can determine how the populations of the different magnetospheric reservoirs (e.g., the plasma sheet and ring current) respond to these changes and with what time delay. In some cases the response could be quite prompt. During the initial $\sim 24$ months portion of the mission, when the Wind spacecraft is in an elliptical orbit which quickly takes it from the solar wind into the magnetosphere, SWICS and STICS will make composition measurements within the magnetosphere which can be compared with solar wind measurements made somewhat earlier and later.

\subsubsection{Fore Shock Region}

The region upstream of the Earth's bow shock and connected to it by the interplanetary magnetic field is known as the fore shock region and is characterized by the frequent presence of accelerated particles and magnetohydrodynamic turbulence. There are two types of upstream ion enhancements which appear to be associated with the bow shock. There is a 'diffuse' component with total energies extending up to several hundred $\mathrm{keV}$ and characterized by a nearly isotropic pitch-angle distribution, and a 'reflected' component which is basically an ion beam with a very narrow spread in both energy (typically 5-10 keV) and pitch angle (peaked along the magnetic field and streaming away from the Earth's bow shock (Paschmann et al., 1981; Ipavich et al., 1981). The reflected component appears to be accelerated at the quasi-perpendicular bow shock while the diffuse component appears 
to be accelerated in the upstream region when there is a quasi-parallel bow shock (Bonifazi and Moreno, 1981). In both cases the solar wind appears to be the source plasma.

For approximately the first two years of the mission the orbit of the WIND spacecraft is optimized for the exploration of the fore shock region. Depending on orbital details, the WIND spacecraft is expected to be in the Earth for shock region about $10 \%$ of the time. Since this portion of the orbit is near spacecraft perigee, WIND will be traveling at relatively high speed through the fore shock. This important aspect of the WIND trajectory will very likely allow us to separate spatial from temporal effects.

SWICS will monitor solar wind charge-state and elemental composition upstream of the bow shock, and STICS will measure these quantities in the accelerated components. Unambiguous charge-state measurements, which SWICS and STICS will provide, are necessary in these events in order to derive particle rigidity, a fundamental parameter in any acceleration theory. SWICS will also measure the kinetic temperature of solar wind ions, which, when correlated with the accelerated component characteristics, will permit comparison with and further refinement of current acceleration models.

In addition to the diffuse and reflected components, magnetospheric particles occasionally appear in the upstream region, characterized by harder spectra, strong anisotropies directed away from the bow shock, and composition characteristic of the mixed plasma in the magnetosphere (Sarris et al., 1978; Möbius et al., 1986). STICS' measurements of the elemental and charge-state composition will allow one to distinguish energetic particles arising from magnetospheric leakage from those arising from in-situ acceleration of a portion of the solar wind.

Measurements of the suprathermal $\mathrm{He}^{+}$upstream of the bow shock are of particular interest. On the one hand it could be the result of magnetospheric leakage, in which case its presence would be correlated with the appearance of magnetospheric $\mathrm{O}^{+}$and $\mathrm{N}^{+}$. On the other hand, the high-thermal speed pick-up $\mathrm{He}^{+}$is very efficiently accelerated by interplanetary shocks (Gloeckler et al., 1994), and presumably also by the bow shock. If this is the case, suprathermal $\mathrm{He}^{+}$will appear along with the high-charge state ions in the diffuse component, which will allow us to study the acceleration of ions with high injection energies.

\subsubsection{Magnetosheath}

During the early portion of the mission, the WIND spacecraft will make multiple passes through the magnetosheath region. This will provide a unique opportunity to make detailed measurements of the shocked solar wind and to investigate the downstream component of upstream events. Because of the high kinetic temperature of the sheath plasma, it has been difficult in the past to make composition measurements in this region. The CHEM instrument on the AMPTE/CCE spacecraft made such measurements (Gloeckler et al., 1989), but because of the orbit, had access to the magnetosheath only during periods of high solar wind pressure. 
SWICS will determine the energy spectra of the various ion species in the sheath under any prevailing solar wind flow conditions. This information is critical in evaluating the probability of solar wind entrance into the magnetosphere.

\subsubsection{Entry of Solar Energetic Particles}

Solar energetic particles (SEP) can be used to determine the gross structure of the magnetosphere. For example, the entry points can define regions where reconnection has occurred between the terrestrial and interplanetary magnetic field. To trace the particle motion it is of course necessary to have detailed measurements of the composition of the energetic particles in interplanetary space. It is important, in particular, to determine mass and ionic charge, and thus the rigidity (momentum/charge), since the behavior of particles with different rigidities can be used to distinguish between various possible entry mechanisms.

STICS will provide the detailed measurements of elemental and ionic charge compositions of solar energetic particles, and of their spectra, in a critical intermediate energy range (10-230 keV/e) which is above that of the solar wind, virtually excluded from entry into the magnetosphere, and below the multi-MeV range solar energetic particles, which appear to have nearly complete access to the outer magnetosphere.

\subsection{STUDIES OF SOLAR PHENOMENA}

The acceleration of the solar wind and of solar energetic particles is a topic of great interest but remains as yet to be fully understood. Solar wind acceleration models require a knowledge of boundary conditions (primarily temperature and density profiles) in the acceleration region and must make predictions that are consistent with all the measured properties of the solar wind at one AU. The SWICS and MASS instruments will provide information about some of the characteristics in the acceleration region. But of even greater importance will be the detailed characterization of the one AU solar wind in terms of composition and charge states, as well as bulk speeds and kinetic temperatures of some 40 ion types. Such measurements of the solar wind properties will play a crucial role in testing and constraining solar wind acceleration models, and should lead to a better understanding of where and how the solar wind is formed.

\subsubsection{Physical Processes $m$ the Solar Corona}

The SMS measurements will provide essential information on the conditions characterizing, and the physical processes operating in that region of the corona where most of the solar wind and solar energetic particle acceleration occur. The solar wind acceleration region lies relatively high in the corona (typically $\sim 1-3$ solar radii in altitude) where the density is very low, especially in coronal holes; hence, spectroscopic measurements of this region are difficult to obtain. As we discuss below, however, an abundance of information regarding the acceleration region is 
available from in situ measurements of solar wind and suprathermal ions. Indeed, it is quite convenient that the very particles whose acceleration and flow are being studied carry much of the necessary information within their composition and behavior.

When the ionization and recombination times characteristic of the dominant ion states of a particular ion species in the solar wind (e.g., the $+6,+7$, and +8 charge states of oxygen, the $+4,+5$, and +6 charge states of carbon, etc.) become long compared with the solar wind expansion time, the ionization state of that species becomes fixed (i.e., 'frozen-in') and remains essentially unchanged as the ions flow through the heliosphere (Hundhausen et al., 1968). The relative abundances of the several ionization states of a particular ion species in the solar wind depend strongly on the electron temperature in the coronal region where the 'freezing-in' occurs. The measured ionization state of solar wind ions from SWICS (and suprathermal ions from STICS) will provide a direct measure of the electron temperature in the region of the corona where the observed ions become frozen-in.

The altitude at which the ionization state of solar wind ions is frozen-in is different for different ion species. For example, under typical coronal conditions, the ionization state of oxygen freezes in near 1.5 solar radii, whereas that of iron is not fixed until $\sim 3$ solar radii. Because the ionization state depends so strongly on the electron temperature where freezing-in occurs, a comparison of the solar wind ionization states of oxygen with those of iron leads to a description of the radial profile of the coronal electron temperature. SWICS will measure not only the ionization state (or charge fraction) of $\mathrm{O}$ and $\mathrm{Fe}$, but also of $\mathrm{C}, \mathrm{Mg}$, and $\mathrm{Si}$, which will provide important information on the coronal temperature and the temperature gradient over a wide temperature range (from less than $10^{6} \mathrm{~K}$ to much greater than $\left.2 \times 10^{6} \mathrm{~K}\right)$.

\subsubsection{Acceleration of the Solar Wind}

The acceleration of heavy ions in the solar wind (helium and $Z>2$ ) is expected to be different from the acceleration of the basic proton-electron plasma. Frictional coupling can be important in the heavy ion expansion (Bürgi and Geiss, 1986). Wave-particle interactions can have a different role in the acceleration of heavy ions than in that of protons (Hollweg, 1978). The composition, temperatures, and mean speeds measured by SWICS of the solar wind heavy ions will reveal the effects of several physical processes important in their expansion. These measurements will provide information concerning the overall proton-electron expansion, for they reveal the nature of the coronal environment in which both the heavy ions and protons are accelerated.

Frictional effects are likely to play an important role in the acceleration of heavy ions, as they serve to couple the heavy-ion expansion to the basic proton-electron expansion. The occurrence of frictional acceleration should be apparent in measurements of the mean bulk speeds of different ion species. If Coulomb interactions are important, ions with large charge-squared-to-mass ratios should show evidence 
of preferential acceleration or deceleration. Other effects can preferentially alter particle speeds. For instance, a polarization electric field arising from electronproton charge separation accelerates heavy ions in proportion to their charge/mass ratio.

Wave-particle interactions and other processes in the corona may selectively increase the temperatures of heavy ions (relative to those of protons and electrons), thus facilitating their escape in the solar wind (e.g., Isenberg and Hollweg, 1983). Such heating processes may be reflected in the kinetic temperatures and mean flow speeds of heavy ions observed in the solar wind. Indeed, earlier solar wind measurements have revealed that ions, at least in selected periods, tend toward equal thermal speeds, leading to an increasing temperature with increasing particle mass (Neugebauer, 1981; Schmidt et al., 1980; Ogilvie et al., 1980). With SWICS we will measure the kinetic temperatures and mean speeds of all major ion species including elements up to iron.

Heavy ions (as well as protons) may be directly accelerated through interaction with a wave field. The resonant acceleration of ions by Alfvén waves has been suggested as a potentially important process (Hollweg, 1978). Such processes may have various dependencies on the ion charge and mass, or no dependence whatsoever. Again, measurements of the mean flow speeds and temperatures of heavy ions in the solar wind should reveal the occurrence of these processes.

\subsubsection{Atom-Ion Separation Processes}

It is well-established that solar energetic particles (SEP) and solar wind elements that have first ionization potentials (FIP) less than $\sim 10 \mathrm{eV}$ are overabundant relative to the corresponding photospheric abundances (Breneman and Stone, 1985; Gloeckler and Geiss, 1989). Therefore, a process seems to be operating that preferentially supplies the corona with elements that are easily ionized. An additional (possibly correlated) mechanism appears to separate ions from neutrals on a time scale corresponding to their ionization rate and to preferentially feed the ionized fraction to the corona. Various models attempting to explain this so-called FIP effect have been proposed (e.g., von Steiger and Geiss, 1989; Geiss and Bürgi, 1986; Vauclair and Meyer, 1985), but none have been completely successful. The breakpoint that separates the 'high' (normal abundance) and 'low' (over abundant) FIP elements appears to be not a sharp step function, but rather a transition region that typically includes the elements carbon, sulfur, and phosphorus. Sometimes this region shifts in FIP to include elements such as oxygen. The magnitude of the relative abundance enhancement of the low FIP elements appears to vary, possibly as a function of solar wind type. For example, the solar wind from coronal holes appears to have only a small FIP effect, having a composition that most closely resembles that of the photosphere (Gloeckler et al., 1989; von Steiger et al., 1992). This could be a consequence of the different solar magnetic field conditions. The SMS experiment will study the temporal variation of the abundance of heavy ions in the solar wind (SWICS and MASS) and SEP events to $\sim 230 \mathrm{keV} / \mathrm{e}$ (STICS), 
which will help in distinguishing among different modeling efforts. In particular, MASS will provide the first continuous measurements of rarer elements and isotopes in the solar wind, including such elements as $\mathrm{Ni}, \mathrm{Ca}, \mathrm{Al}$, and $\mathrm{Na}$ with low FIP $(<8 \mathrm{eV})$ and $\mathrm{S}$ and possibly $\mathrm{P}$ with a FIP in the transition region.

\subsubsection{Isotopic Composition of the Solar Wind}

Current measurements of solar wind isotopes heavier than helium are restricted, with a majority coming from lunar sample analysis and the foil collection technique (Geiss et al., 1972). SWICS and STICS will provide isotopic measurements for helium $\left({ }^{3} \mathrm{He}\right.$ and $\left.{ }^{4} \mathrm{He}\right)$ in the solar wind, for low energy SEPs and interstellar helium. With the MASS sensor we will obtain for the first time a continuous record of isotopic abundances of solar wind heavy ions (e.g. $\mathrm{Ne}, \mathrm{Mg}$, and $\mathrm{Si}$ ). Solar wind isotopic abundance measurements with MASS will also permit for the first time a direct correlation with simultaneous SEP isotopic measurements (by the energetic particle experiment on WIND), which may resolve apparent disagreements in, e.g., the ${ }^{20} \mathrm{Ne} /{ }^{22} \mathrm{Ne}$ ratio (Geiss et al., 1972; Gloeckler and Geiss, 1989).

\subsubsection{Coronal Transients}

Coronal mass ejection (CME) events appear to originate in (initially) magnetically closed or quasi-closed coronal regions (e.g., Burlaga, 1984). The mass ejections from such regions move outward from the sun to form a small but not insignificant part of the solar wind. (Flare-associated interplanetary shocks are the most dramatic solar wind manifestation of coronal transients in the low-latitude inner heliosphere) As noted in the preceding sections, solar wind heavy ion measurements provide important information regarding the properties of the coronal region in which the wind originates. Hence, measurements by SWICS and MASS of heavy ions in solar wind plasma ejected as a coronal transient will give information regarding the properties of magnetically closed coronal regions, perhaps yielding important clues to the mechanism(s) whereby mass-ejection coronal transients are driven (e.g., Galvin et al., 1987). The interplanetary manifestations of these magnetically (quasi) closed structures sometimes include the bi-directional streaming of suprathermal ions (Marsden et al., 1987), which would be measured by STICS.

\subsubsection{One AU Baseline Measurements}

A SWICS sensor (Gloeckler et al., 1983, 1992) is successfully flying on the Ulysses spacecraft and is currently making detailed measurements of the elemental and ionic-charge composition and flow properties of solar wind and interstellar pick-up ions at all heliographic latitudes (Gloeckler et al., 1993). (Ulysses was launched October 1990 and began the out-of-the- ecliptic phase of the mission in February 1992. The first polar pass takes place in 1994.) A comparison of solar wind elemental and ionic charge compositions at two different latitudes, and of the coronal conditions they imply, will be made using data from the two similar SWICS sensors on the two spacecraft. We will use these simultaneous measurements from 
WIND (in the ecliptic) and Ulysses (out of the ecliptic) to help separate spatial and temporal variations and thus to determine how coronal conditions and processes vary with heliographic latitude.

\subsubsection{Solar Particle Acceleration}

The elemental and particularly the ionic charge composition of particles accelerated in solar energetic particle (SEP) events can provide detailed information on the flare injection and acceleration processes, and on the locations where these processes occur. It is known that particles accelerated in small flare events can have highly unusual abundances. These events can contain more ${ }^{3} \mathrm{He}$ than ${ }^{4} \mathrm{He}$; they can have more $\mathrm{Fe}$ than $\mathrm{O}$; they can contain substantially more $\mathrm{O}$ than $\mathrm{C}$. These compositional anomalies may be the result of a preferential injection process. The unambiguous measurements with STICS of the charge states of particles accelerated in small flare events compared to regular flare events will provide a critical test of existing models. For example, in one model (Fisk, 1978) the charge states of elements with unusual abundances should be restricted to those ions that can interact effectively with excited electrostatic ion-cyclotron waves. The only charge state of $\mathrm{O}$ that satisfies this constraint is +5 ; for $\mathrm{Fe}$ the preferred charge state is +16 or +17 . If it can be established that preferential injection mechanisms are operative in flare events, this result will place strong constraints on acceptable conditions at the flare site for different models. For instance, the mechanism of Fisk requires that the ratio of thermal-to-magnetic energy is very low, the electron-to-ion-temperature ratio is not excessive, and that the currents are relatively weak.

As is the case for the solar wind, the charge states of SEP events are essentially determined at the acceleration site, where they are 'frozen-in'. Measurements by STICS of the charge states of the ions accelerated in flares will constrain the temperature and thus the location of the region where the acceleration process occurs. If the acceleration occurs in the main flare region, where the plasma is being rapidly heated, we might expect a high degree of ionization for the accelerated particles. In contrast, if the acceleration occurs in the surrounding corona, such as by a shock wave triggered by the flare, we should expect charge states more characteristic of average coronal conditions, i.e., of a plasma with an electron temperature $\sim 1.5 \times 10^{6} \mathrm{~K}$.

Important constraints on the rigidity and/or energy dependence of the flareacceleration process can be obtained from the spectra of the accelerated particles; e.g., the spectra which result from a shock mechanism will differ from those which result from a statistical process. In interpreting the spectral data it is necessary to know the particle charge-to-mass ratios, and thus their rigidity. Heavier ions at low energies will be only partially stripped of their electrons, and thus a variety of charge-to-mass ratios will occur. With SWICS at low energies and with STICS at high energies we can measure uniquely the mass, the ionic charge and the energy spectra of all major ion species from $\mathrm{H}$ through $\mathrm{Fe}$, over an energy range from solar-wind energies to $\sim 230 \mathrm{keV} /$ charge. 


\subsection{STUDIES OF HELIOSPHERIC PHENOMENA}

\subsubsection{Particle Acceleration}

Particles are accelerated in the solar wind up to energies $\sim 1 \mathrm{MeV} \mathrm{nucl}^{-1}$ by propagating shock waves that are generated by solar flares. They are also accelerated, particularly in solar-minimum conditions, in association with stream-stream interaction regions in the solar wind (Tan et al., 1989; Reames et al., 1991). During solar minimum conditions, the Sun produces relatively steady high-speed solar wind streams, which, upon interacting with lower-speed wind at several AU from the Sun, accelerate particles that propagate back toward Earth in steady corotating particle streams. Each of these examples of acceleration is important to study since they can provide, by analogy, information on particle acceleration in large-scale astrophysical plasmas in less accessible regions of the Universe.

Unambiguous measurements of the mass and the ionic charge, and thus of the rigidity, of the accelerated particles, and of their spectra over a wide energy range, can give answers to presently unsolved problems associated with the acceleration, viz., the source of the accelerated particles and the nature of the acceleration.

Particles accelerated in the solar wind could come directly from the solar wind plasma, or they could originate as more energetic particles of solar origin (such as high velocity tails of the solar wind), or as pick-up ions. If the former occurs, the requirements on the acceleration process are of course more severe. With the capability of SWICS and STICS to measure uniquely the mass and ionic charge of the solar wind and suprathermal ions, we can constrain the likely source of the accelerated particles. If the origin is the solar wind, the accelerated particles must have solar wind charge states; in contrast, if they originate as more energetic particles they could exhibit a different degree of ionization. Indeed, if the source of the accelerated particles would be the local solar wind plasma, an exact correspondence between the charge states of the thermal and the accelerated particles would be required. Finally, accelerated pick-up ions are easily distinguished from solar wind ions by their low charge state (e.g., $\mathrm{He}^{+}$).

As with solar flares, the nature of acceleration processes in the solar wind is revealed in the rigidity and/or energy dependence of the spectra that they produce. STICS, with its capability to measure the mass, the ionic charge and the spectrum of ion species from $\mathrm{H}$ to $\mathrm{Fe}$, will provide detailed constraints on these dependencies. The SMS spectral measurements cover the energy range from solar wind energies up to $\sim 230 \mathrm{keV} /$ charge; i.e., they span the energy range from where the particles are injected into the acceleration process up to some of the higher energies obtained in typical events.

\subsubsection{Comparisons with Ulysses}

The extent to which, and the means by which particles are accelerated in the solar wind should vary markedly with heliographic latitude. For example, the strength of stream-stream interaction regions in the solar wind, which produce corotating 
streams of accelerated particles, should diminish with latitude as solar rotation effects become less important. A comparison of energetic particle measurements from Ulysses and from WIND will reveal how the acceleration processes vary with latitude in general, and perhaps in specific events. Steady solar wind streams often originate from coronal holes, and thus the acceleration they produce could extend over a wide latitude range and be observed simultaneously by SWICS on Ulysses and WIND. Similarly, shock waves from large flare events, and possibly CMEs could have a broad latitude extent. We should be able to compare measurements made with STICS on WIND with energetic particle measurements of energetic particles (Lanzerotti et al., 1992) being made on Ulysses.

\subsubsection{Plasma Processes in the Solar Wind}

The SMS experiment also provides the opportunity to investigate a variety of interesting plasma processes in the solar wind. For example, there can be local heating of the solar wind at propagating shock waves or in stream-stream interaction regions; there can be instabilities driven by heat flux and other means. SWICS, with its capability of measuring the kinetic temperatures of all major solar wind ions, will provide an important probe of these processes. The dominant instabilities occurring can be revealed in the response of the kinetic temperatures of solar wind ions with different charge/mass ratios. As a simple example, when the ions cyclotron-damp the instabilities, the cyclotron frequency of the ions that absorb the most energy reveals the dominant frequency excited. Spectral measurements by SWICS and STICS will also reveal when and how plasma processes generate extended suprathermal tails on the solar wind ion distributions.

\subsubsection{Detailed Studies of Pick-up Ions}

Pick-up ions are produced wherever there is a source of neutral molecules or atoms. Ionized by solar UV and/or the solar wind, these newly formed ions are immediately accelerated by the $\mathbf{V}_{s w} \times \mathbf{B}$ electric field, gyrating about the ambient $\mathrm{B}$ field, thus forming a 'ring'-type distribution in velocity space at $V=V_{s w}$ in the solar wind reference frame. Because of variations in the direction of $\mathbf{V}_{s w}$ and $\mathbf{B}$ and rapid pitch-angle scattering this ring distribution quickly spreads into a 'shell' distribution. Adiabatic deceleration in the expanding solar wind fills in the velocity space for $V<V_{s w}$ to a degree that depends on the spatial distribution of neutrals between the Sun and the observation point. Energy diffusion and acceleration to speeds beyond Vsw has been predicted (e.g., Isenberg, 1991).

Three types of pick-up ions have been observed so far: (a) cometary pick-up ions (e.g., Ipavich et al., 1986; Gloeckler et al., 1986), (b) interstellar pick-up ions (Möbius et al., 1985; Gloeckler et al., 1993), and (c) lunar pick-up ions (Hilchenbach et al., 1991, 1992). Using data obtained with the STICS instrument on WIND it will be possible to study in great detail the 3-dimensional distribution functions of interstellar pick-up helium throughout the duration of the mission and of lunar pickup ions especially during the lunar swingby portion of the mission. 
The interstellar helium studies are likely to prove significant because it will be possible to examine the physics of the pick-up process in great detail. In addition, the longitudinal distribution of interstellar neutral helium will be mapped using measurements of the fluxes of pick-up $\mathrm{He}^{+}$and solar EUV flux provided by SOHO. This information, along with measurements of pick-up $\mathrm{He}^{+}$made with SWICS on Ulysses, will allow us to infer the 3-dimensional distribution of interstellar helium in the inner heliosphere, and to determine the ionization (loss) rate of neutral helium. It should also be possible to measure with sufficient precision the ${ }^{3} \mathrm{He} /{ }^{4} \mathrm{He}$ ratio in the local interstellar gas, a value of great importance for models of cosmology.

\section{Instrument Description}

In SWICS we combine electrostatic deffection with post-acceleration, followed by a time- of-flight (TOF) and energy measurement. SWICS is a modified version of an instrument that has flown and worked successfully on AMPTE/CCE and more recently on Ulysses. STICS uses electrostatic deflection analysis, followed by a time-of-flight and energy measurement. Post-acceleration is not necessary since the ions measured by STICS have energies above the threshold of the low-noise solid-state detector in the time-of-flight system. An identical STICS instrument is part of the EPIC investigation (Williams et al., 1993) on the Geotail spacecraft, and is currently operating and returning data as planned. MASS uses electrostatic deflection followed by a time-of-flight measurement but does not require an energy measurement and therefore does not have solid-state detectors.

The sensors determine separately the mass $(M)$, mass per charge $(M / Q)$, and the energy $(E)$ of individual ions in the solar wind (SWICS), and of individual ions with suprathermal energies up to $230 \mathrm{keV} / \mathrm{e}$ (STICS). High mass resolution measurements are available at solar wind energies from MASS. For reference, a summary of the instrument capabilities is given in Table I.

A common Data Processing Unit (DPU) is shared by the SWICS, STICS, and MASS sensors, and is described in Section 3.4.

\subsection{THE SOLAR WIND ION COMPOSITION SPECTROMETER (SWICS)}

\subsubsection{Principle of Operation of SWICS}

The SWICS sensor is based on techniques of particle identification using a combination of electrostatic deflection, moderate post-acceleration, time-of-flight and energy measurement (Gloeckler, 1977; Gloeckler and Hsieh, 1979). Figure 1 shows schematically the principle of operation of the SWICS sensor and illustrates the function of the 5 basic sensor elements used:

(1) Ions of kinetic energy $E$, mass $M$, and charge (ionization) state $Q$ enter the sensor through a large area, multi-slit collimator that selects proper entrance trajectories of the particles. 
TABLE I

Instrument capabilities

\begin{tabular}{|c|c|c|c|}
\hline & $\begin{array}{l}\text { SWICS } \\
\text { sensor }\end{array}$ & $\begin{array}{l}\text { MASS } \\
\text { sensor }\end{array}$ & $\begin{array}{l}\text { STICS } \\
\text { sensor }\end{array}$ \\
\hline Ion species & $\mathrm{H}-\mathrm{Fe}$ & $\mathrm{He}-\mathrm{Ni}$ & $\mathrm{H}-\mathrm{Fe}$ \\
\hline Mass/charge range (amu/e) & $1-30$ & - & $1-60$ \\
\hline Energy range $(\mathrm{keV} / \mathrm{e})$ & $0.5-30$ & $0.5-11.6^{\mathrm{a}}$ & $8-226$ \\
\hline \multicolumn{4}{|l|}{ Mean speed range $\left(\mathrm{km} \mathrm{s}^{-1}\right)$} \\
\hline $\mathrm{H}^{+}$ & $310-2400$ & - & - \\
\hline $\mathrm{O}^{+6}$ & $190-1470$ & $200-900$ & - \\
\hline $\mathrm{Fe}^{+10}$ & $130-1010$ & $200-500$ & - \\
\hline \multicolumn{4}{|l|}{ Resolution (FWHM) } \\
\hline Energy, $\Delta(E / Q) /(E / Q)$ & 0.06 & 0.05 & 0.05 \\
\hline Mass/charge, $\Delta(M / Q) /(M / Q)$ & 0.04 & - & 0.15 \\
\hline Mass, $\Delta M / M$ & $0.2^{\mathrm{a}}$ & $0.01^{\mathrm{a}}$ & $0.12^{b}$ \\
\hline \multicolumn{4}{|l|}{ Total instrument geometrical factor } \\
\hline $\mathrm{cm}^{2} \mathrm{sr}$ & $2.3 \times 10^{-3}$ & - & 0.05 \\
\hline $\mathrm{cm}^{2}$ & $1.8 \times 10^{-2}$ & 0.35 & - \\
\hline \multicolumn{4}{|l|}{ Field of View (FOV) } \\
\hline Pointing direction is & $22.5^{\circ} \mathrm{wrt}$ & $22.5^{\circ} \mathrm{wrt}$ & $0^{\circ}$ \\
\hline perpendicular to spin axis & STICS & STICS & \\
\hline FOV is fan-shaped & $4^{\circ}$ by $45^{\circ}$ & $4^{\circ}$ by $40^{\circ}$ & $4.5^{\circ}$ by $156^{\circ}$ \\
\hline Dynamic range & $10^{10}$ & $10^{10}$ & $5 \times 10^{10}$ \\
\hline \multicolumn{4}{|l|}{ Minimum flux } \\
\hline$\left(\mathrm{cm}^{2} \mathrm{ssr} \mathrm{keV/e}\right)^{-1}$ & - & $10^{-6}$ & \\
\hline$\left(\mathrm{cm}^{2} \mathrm{~s}\right)^{-1}$ & $10^{-2}$ & $10^{-2}$ & - \\
\hline
\end{tabular}

(2) The electrostatic deffection analyzer serves both as a UV trap and an energy per charge $(E / Q)$ filter, allowing only ions within a given energy per charge interval (determined by a stepped deflection voltage) to enter the time-of-flight vs energy system.

(3) Ions are post-accelerated by up to $30 \mathrm{kV}$ potential drop just before entering the time-of-flight vs energy system. The energy they gain is sufficient to be adequately measured by the solid state detectors which typically have a 25 to $35 \mathrm{keV}$ energy threshold. An energy measurement is required for determining the mass composition of an ion population, and ions with energies below $\sim 30 \mathrm{keV}$ must be accelerated if their mass is to be identified.

(4) In the time-of-flight (TOF) system the speed of each ion is determined by measuring the travel time $\tau$ of the particle between the start and stop detectors separated by a dictance of $10.5 \mathrm{~cm}$. 


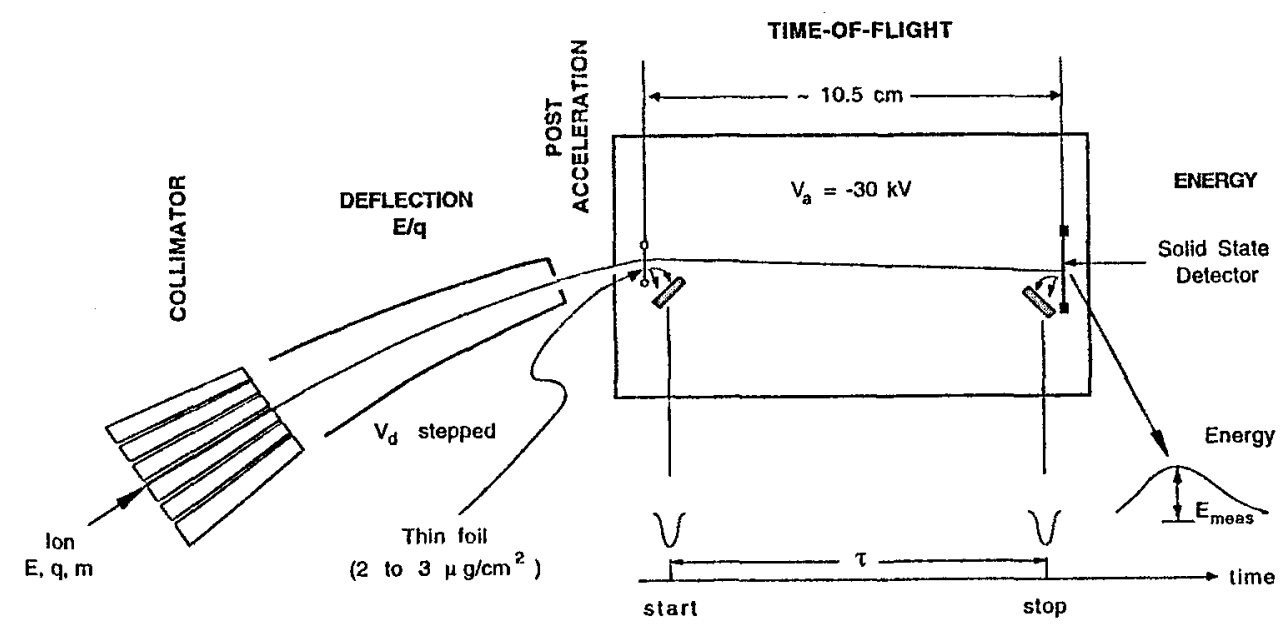

Fig. 1. Schematic of the measurement technique used in SWICS showing the functions of each of the basic elements.

(5) The particle identification is completed by measuring the residual energy of the ions in a conventional low-noise solid-state detector.

From simultaneous measurements of the time-of-flight, $\tau$, and residual energy, $E_{\text {meas }}$, and a knowledge of $E / Q$ and the post-acceleration voltage, $V_{a}$, we can determine the mass $(M)$, charge state $(Q)$, incident energy $(E)$, or incident speed $\left(V_{\text {ion }}\right)$ of each ion as follows:

$$
\begin{array}{ll}
M=2(\tau / d)^{2}\left(E_{\text {meas }} / \alpha\right) & Q=\left(E_{\text {meas }} / \alpha\right) /\left(V_{a}+E^{\prime} / Q\right) \\
M / Q=2(\tau / d)^{2}\left(V_{a}+E^{\prime} / Q\right) & E=Q \cdot(E / Q) \quad V_{\text {ion }}=438 \cdot(E / M)^{1 / 2},
\end{array}
$$

where $d$ is the flight path, $E^{\prime} / Q$ takes account of the small energy loss of ions in the thin foil of the start-time detector and $\alpha$ is the nuclear defect in solid state detectors (Ipavich et al., 1978). The units of $V_{\text {ion }}$ are $\mathrm{km} \mathrm{s}^{-1}$ when $E / M$ is in $\mathrm{keV} / \mathrm{amu}$.

\subsubsection{Description of the SWICS Instrument}

The SWICS instrument will determine the energy as well as the elemental and ionic charge state composition of all major solar wind ions from $\mathrm{H}$ through $\mathrm{Fe}$. The simplified side cross sectional view of the SWICS sensor is shown in Figure 2 and the associated electronic block diagram is given in Figure 3. A similar SWICS instrument on Ulysses has been fully described by Gloeckler et al. $(1983,1992)$.

The SWICS sensor (see Figure 2) consists of a cylindrical inner container divided into 3 compartments and a larger co-axial cylindrical outer housing to which the electrostatic deflection analyzer is attached. The three inner compartments are kept at a high potential (typically $-30 \mathrm{kV}$, but commandable to lower levels) by the Post Acceleration Power Supply (PAPS) which is housed in its own cylindrical container (not shown in Figure 2). The inner compartments are supported entirely 


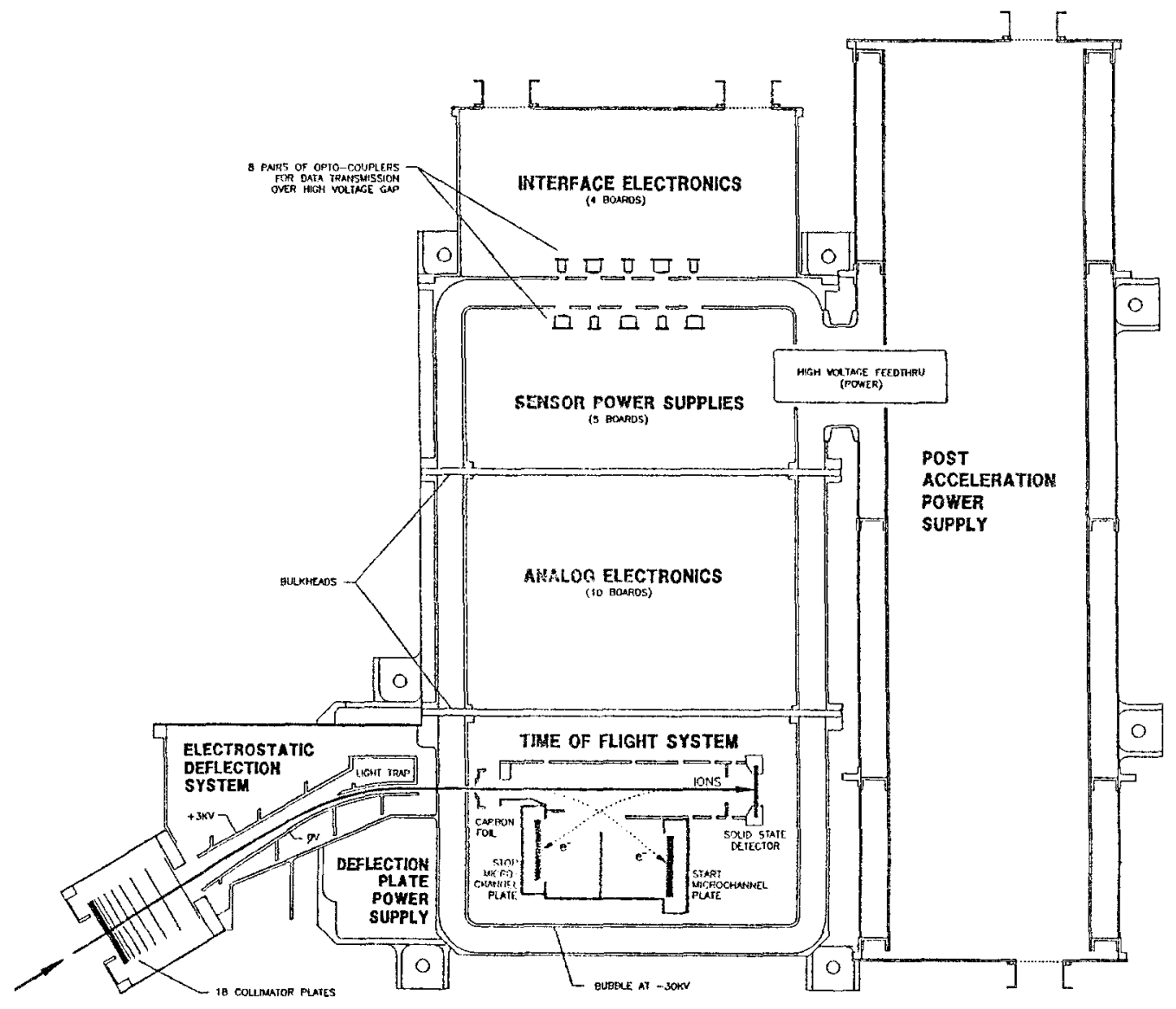

ION

Fig. 2. Simplified side cross sectional view of the SWICS sensor. The $-30 \mathrm{keV}$ post-acceleration supply is not shown.

by two insulator bulkheads, one of which also physically isolates the ultra-clean time-of-flight vs energy section from the analog electronics. The other bulkhead supports the sensor power supply and optocoupler system for which separate venting is provided. A $10 \mathrm{~mm}$ gap and vapor-deposited parylene coating on facing surfaces (except for the TOF section outer housing) provide high voltage isolation between the inner and outer housings. Power from the PAPS is supplied to the high voltage bubble by means of an isolation transformer through a multi-pin high voltage feed-through connected to the upper compartment. Digital signals are transferred across the $10 \mathrm{~mm}$ vacuum gap by eight optocouplers.

3.1.2.1. SWICS Deflection Analyzer Ions enter the electrostatic reflection analyzer system through a large area, conical, muiti-slit collimator. The width of the 
individual channels in the collimator are such as to limit dispersions in the analyzer and flight path differences in the time-of-flight system to $<0.5 \%$. The deflection system has a small angle, conical configuration with pie- shaped $45^{\circ}$ deflection plates. The deflection plates are connected to a variable voltage supply which is housed immediately above the deflection system and which increments the deflection voltage in 60 logarithmic steps (one step per spin, i.e., $\sim 3 \mathrm{~s}$ ) separated by $7 \%$. Serration, black-coating, and light traps are used to effectively eliminate reflection of visible and UV radiation into the time-of-flight system.

The deflection analyzer has a $6 \%$ energy/charge resolution and is used for the full mass vs mass per charge analysis of solar wind ions and their high velocity tails in the range 0.5 to $30 \mathrm{keV} /$ charge. The time-of-flight vs energy system is placed behind the exit slit of the analyzer and inside the high voltage bubble. At any given voltage step the analyzer passes ions which have equal (to within the $6 \%$ resolution) energy per charge. These ions are then post-accelerated, and their time-of-flight and total residual kinetic energy are measured by the time-of-flight vs energy system.

\subsubsection{SWICS Time-of-Flight System The SWICS time-of-flight vs ener-} gy assembly consists of a 'start' and a 'stop' detector separated by $10.5 \mathrm{~cm}$. A schematic representation of the time-of-flight system is shown in Figure 1. The start and stop signals are derived from secondary electrons which are released with a mean energy of a few $\mathrm{eV}$ when an energetic ion enters or leaves a solid surface. The surface material used for the start detector is that of a thin, $\sim 2 \mu \mathrm{g} \mathrm{cm}{ }^{-2}$ carbon foil (supported on a $85 \%$ transmission nickel grid), and for the stop detector that of the gold front surface of the Au-Si solid state detector. The secondary electrons from the start and stop detectors are accelerated to $1 \mathrm{kV}$ and then deflected by a system of acceleration gaps and deflection surfaces and strike the respective microchannel plate (MCP) assembly. A common supply $(1 \mathrm{kV})$ is used to both accelerate and deflect the electrons. The microchannel plates are normally biased at $2 \mathrm{kV}$ to operate at a gain of $5 \times 10^{6}$. This bias voltage (and hence the gain) is adjustable by ground command. The slight difference in the flight path of the secondary electrons introduces a timing uncertainty of less than the $0.5 \mathrm{~ns}$ FWHM resolution of the analog electronics. Ions are practically unaffected by the electric fields of the time-of-flight assembly because of their higher energy.

The residual energy is measured by one of three low-noise rectangular $\mathrm{Au}-$ Si solid-state detectors. The center detector is used for the analysis of the bulk (thermal) solar wind plasma when the instrument points (once per spin) in the solar direction and for non-thermal plasmas in all other directions. The two side detectors will measure primarily non-thermal plasmas. Together the three detectors have a combined $45^{\circ}$ field of view, and by sectoring their respective outputs we will obtain anisotropy information above, below, and in the ecliptic plane. 


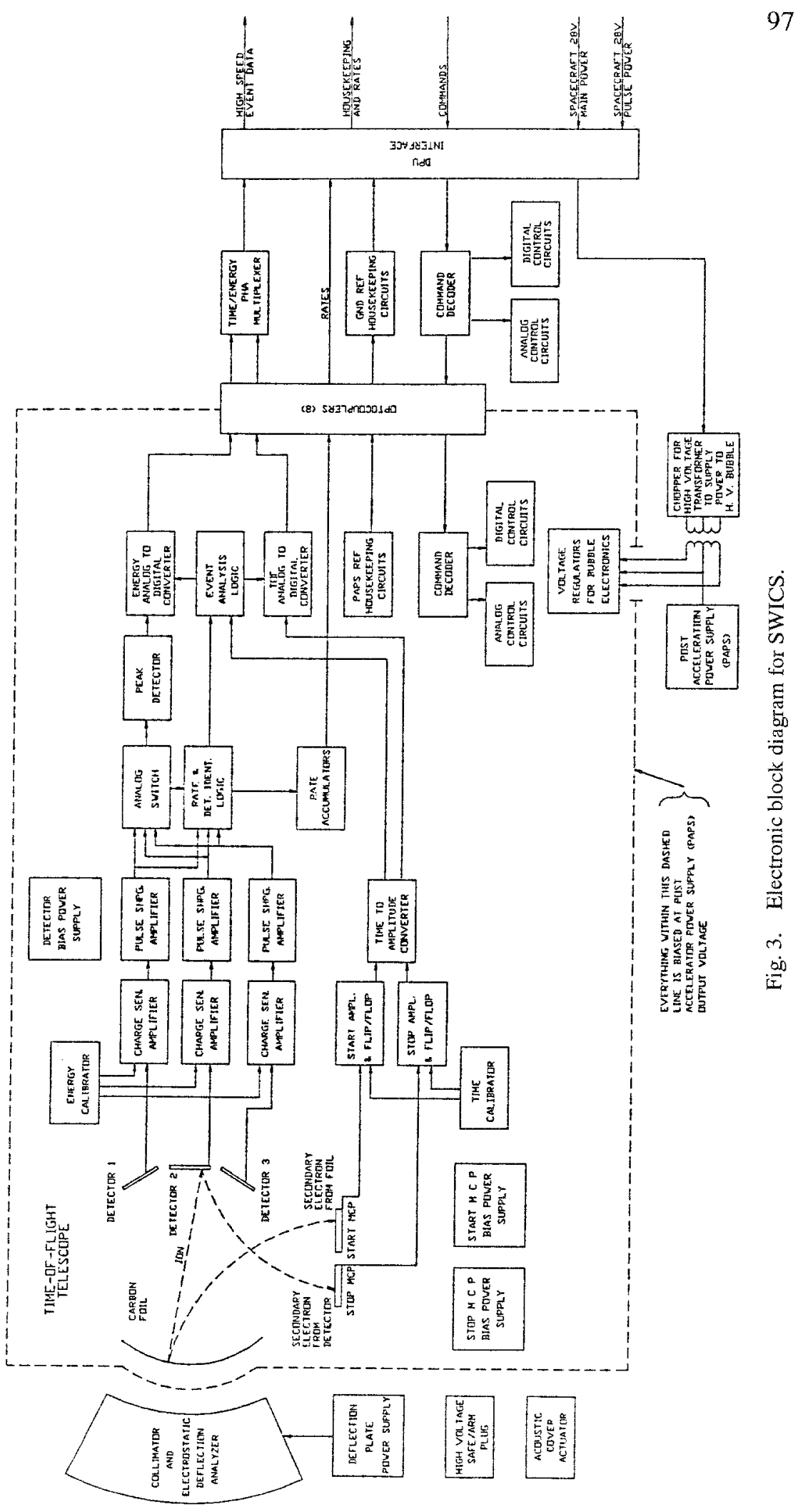




\subsection{THE SUPRA-THERMAL ION COMPOSITION SPECTROMETER (STICS)}

The STICS sensor will complement SWICS by measuring the 3-dimensional distribution functions of supra-thermal ions and energetic particles up to energies of $\sim 230 \mathrm{keV} /$ charge. This instrument, like SWICS, will determine the mass/charge (above $\sim 8 \mathrm{keV} / \mathrm{e}$ ) and mass (above $\sim 30 \mathrm{keV} / \mathrm{e}$ ) of ions, their arrival directions both in and out of the ecliptic plane, and their energy spectra. Because the fluxes of suprathermal heavy ions are generally quite low, STICS has a large geometrical factor $\left(0.05 \mathrm{~cm}^{2} \mathrm{sr}\right)$ which is essential for statistically meaningful analyses of the charge state and elemental composition in upstream bursts and co-rotating particle streams and for comprehensive measurements of the charge states of a number of key elements in events associated with solar flares and interplanetary acceleration (e.g., energetic storm particles). This large geometrical factor and the full 3-dimensional capabilities of STICS are also essential for detailed studies of pick-up ions.

\subsubsection{Principle of Operation of STICS}

The principle of operation of STICS is the same as for SWICS (see Section 3.1.1). However, since STICS is designed to measure the mass and mass/charge composition of supra- thermal ions above the upper energy limit of SWICS $(30 \mathrm{keV} / \mathrm{e})$, no post-acceleration after the electrostatic deflection analyzer is used or required.

\subsubsection{Description of the STICS Instrument}

Physically, the STICS instrument is made up of two units: the STICS Sensor and the STICS Analog Electronics box. The sensor contains the deflection system, three time-of-flight telescopes (including three solid state detectors for the energy measurements), the high voltage power supplies for the deflection analyzer system and the microchannel plates, and the detector preamplifiers. The remainder of the analog electronics, including shaping amplifiers, discriminators, time-of-flight circuits, the analog-to-digital converters (ADCs), and the valid-event logic (for the pulse height data), as well as the low voltage power supply, are located in the separate Analog Electronics Box. This is done to free the mechanical design of these circuits from the constraints imposed by the odd shape of the sensor package. Outputs from the Analog Electronics box pass into the common Data Processing Unit where they are processed for transmission to the spacecraft. A simplified schematic cross section of the STICS sensor and a block diagram of the STICS electronics are given in Figures 4 and 5, respectively.

3.2.2.1. STICS Deflection Voltage System Particles enter the STICS electrostatic deflection analyzer system through a simple entrance aperture that includes a light trap. Mechanically, the STICS electrostatic deflection system consists of two concentric spherical segments with the following characteristics: 
- Polar acceptance angle:

$159^{\circ}$

- Mean radius $\left(R_{0}\right)$ :

$107.75 \mathrm{~mm}$

- Gap $(\Delta R)$ :

$4.50 \mathrm{~mm}$

- Deflection angle:

$125^{\circ}$

- Number of output voltages:

32 per hemisphere

- Number of voltage steps:

30

- Voltage range (+outer/-inner):

0.263 to $9.45 \mathrm{kV}$

- Dynamic range:

- Step spacing:

36 per science record

$-E / Q$ range:

$12.25 \%$

- $E / Q$ resolution:

6.30 to $226.3 \mathrm{keV} / \mathrm{e}$

$\sim 5 \%$, FWHM

- Voltage modes:

3 pre-defined cycles

1 commandable cycle

freeze voltage cycle

The outer and inner deflection plates of the electrostatic deflection system are connected respectively to the positive and negative high voltage deflection plate power supplies (+DPPS and -DPPS) which set the deflection voltages of both plates simultaneously. There are thirty-two output voltages for each plate. These voltages are logarithmically spaced to take the plates from $\pm 260 \mathrm{~V}$ to the maximum deflection voltage of $+10 \mathrm{kV}$ in order to cover the entire energy-per-charge $(E / Q)$ range of the sensor ( $\sim 10$ to $230 \mathrm{keV} / \mathrm{e})$.

The settings of the +DPPS and the -DPPS are represented by their respective step numbers. The + DPPS and the -DPPS will have the same step number (matched positive and negative voltages) at any given time. However, the \pm DPPS are enabled/disabled independently in case there is a partial failure in one of the power supplies. For a given step number $n$, where $n=0$ to 31 and both \pm DPPSs are enabled, the energy per charge response (neglecting fabrication tolerances and fringe field effects) is given by

$$
E / Q=10.0 \times(2.0000)^{[(n-4) / 6]}, \text { where } n=0 \text { to } 31 \text { (step number). }
$$

The $E / Q$ values of the voltage steps are used in the DPU for the Mass per Charge classification of event words. If one of the DPPS's is disabled, the DPU adjusts the $\mathrm{E} / \mathrm{Q}$ values accordingly (i.e., the estimated $E / Q$ value is halved).

The DPPS steps once every one or two spins (i.e., every 3 or $6 \mathrm{~s}$ ) depending on the telemetry rate. The voltage step cycle period consists of 30 step periods (to synchronize with the SMS science record, which is contained in a 60 spin interval). There are three pre-defined voltage step cycles (see the Fine, Medium and Fast Modes in Table II). In the commandable cycle, any of the 30 slots can be allocated by command to any combination of the available 32 voltage steps. In addition, 


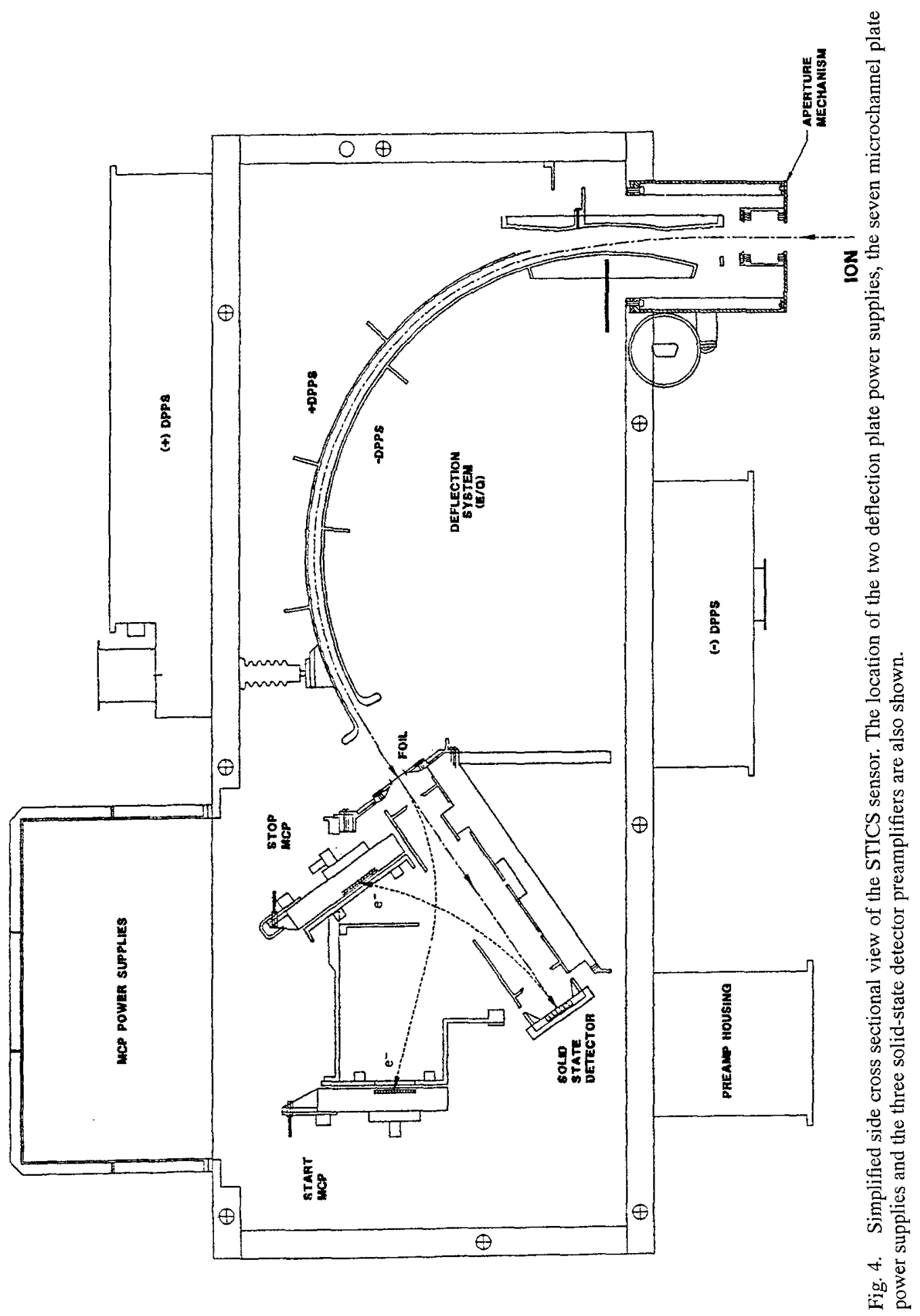




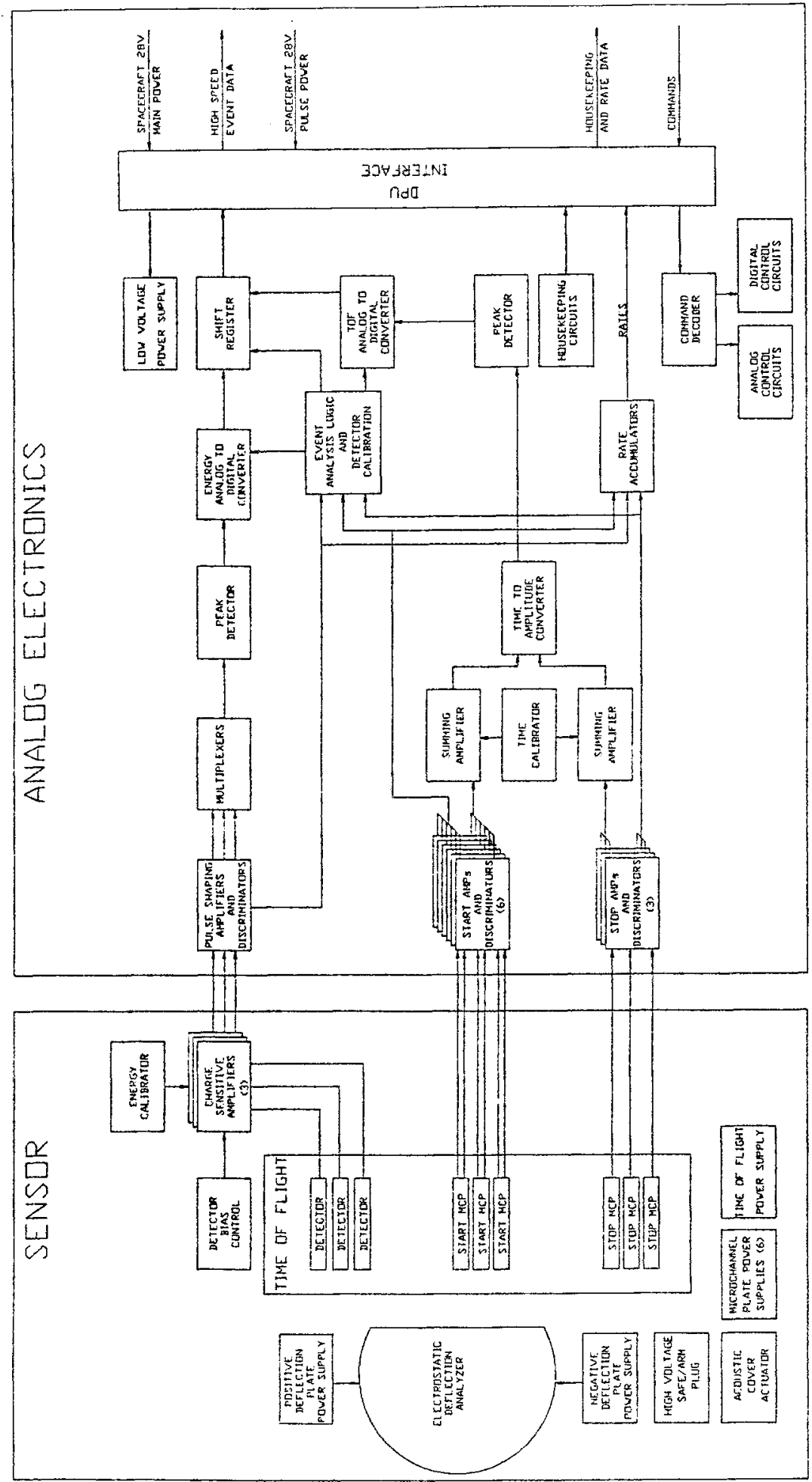

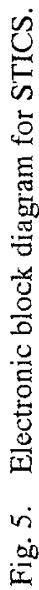


a two-command sequence consisting of a 'freeze the voltage step' command and another command specifying the desired voltage step number allows one to hold the deflection voltage at the specified step value throughout the voltage cycle.

The default voltage cycle used for flight consists of a repeated 10 step subsequence, shown below as the Fast Mode, covering the energy/charge range $230 \mathrm{keV} / \mathrm{e}$ to $\sim 10 \mathrm{keV} / \mathrm{e}$ at $\sqrt{2}$ intervals. The 10-step sequence is repeated 3 (6) times during an SMS science record in the low (high) bit rate mode.

In low bit rate (e.g., halo orbit), each voltage step period consists of two spacecraft spin periods (nominally $6 \mathrm{~s}$ ). In high bit rate (i.e., near Earth), the voltage step period equals the spin period (nominally $3 \mathrm{~s}$ ).

\subsubsection{STICS Time-of-Flight System The STICS time-of-flight vs energy system} is conceptually the same as that described earlier for SWICS. Particles with the correct E/Q make it through the deflection system and strike the thin $\left(\sim 2 \mu \mathrm{g} \mathrm{cm}^{-2}\right)$ carbon foil at the entrance of the time-of-flight telescope. Secondary electrons are knocked off the inner surface of the foil, while the incoming particles pass relatively undisturbed into and through the time-of-flight telescope chamber. The secondary electrons are deflected by electric fields inside the telescope and strike one of the three START microchannel plates, generating the start signal for the time-of-flight analysis. The deflection preserves the position of origin of these electrons as they strike the microchannel plates. Six discrete anodes (two per microchannel plate) lie behind the START signal microchannel plates, providing information on the entrance angle (six equal polar sectors covering $+80^{\circ}$ to $-80^{\circ}$ ) of the incoming particles. At the far end of the time-of-flight telescope, the incoming particles strike one of the three solid state detectors. Secondary electrons from the front surface of the solid state detectors are deflected onto three STOP microchannel plates, providing the stop signal for the time-of-flight analysis. The solid state detectors also provide energy information on the incident particles. The energy and time-of-flight information is transmitted to the DPU in the form of pulse height event words, which are used in the mass and mass-per-charge classification by the DPU.

\subsection{THE HIGH-RESOLUTION MASS SPECTROMETER (MASS)}

The MASS sensor will determine the elemental and isotopic composition of solar wind ions using a new application of the time-of-flight measurement technique that results in exceptionally high mass resolution $(M / \Delta M>100)$. The high mass resolution of the MASS sensor allows for the first time the measurement of the abundances of the rarer solar wind species, including some elements heavier than iron and many isotopic ratios. The MASS sensor consists of an electrostatic deflection system, a time-of-fiight (TOF) mass analyzer system, associated high voltage supplies and a low voltage power converter, and analog and digital electronics. A simplified cross section and block diagram of the MASS sensor are given in Figures 6 and 7, respectively. 
TABLE II

STICS pre-defined voltage cycle modes

\begin{tabular}{|c|c|c|c|}
\hline $\begin{array}{l}\text { Step } \\
\text { No. }\end{array}$ & $\begin{array}{l}\text { Fine mode } \\
(\mathrm{keV} / \mathrm{e})\end{array}$ & $\begin{array}{l}\text { Medium mode } \\
(\mathrm{keV} / \mathrm{e})\end{array}$ & $\begin{array}{l}\text { Fast mode } \\
(\mathrm{keV} / \mathrm{e})\end{array}$ \\
\hline 31 & 226.3 & 226.3 & 226.3 \\
\hline 30 & 201.6 & & \\
\hline 29 & 179.6 & 179.6 & \\
\hline 28 & 160.0 & & 160.0 \\
\hline 27 & 142.5 & 142.5 & \\
\hline 26 & 127.0 & & \\
\hline 25 & 113.1 & 113.1 & 113.1 \\
\hline 24 & 100.8 & & \\
\hline 23 & 89.8 & 89.8 & \\
\hline 22 & 80.0 & & 80.0 \\
\hline 21 & 71.3 & 71.3 & \\
\hline 20 & 63.5 & & \\
\hline 19 & 56.6 & 56.6 & 56.6 \\
\hline 18 & 50.4 & & \\
\hline 17 & 44.9 & 44.9 & \\
\hline 16 & 40.0 & & 40.0 \\
\hline 15 & 35.6 & 35.6 & \\
\hline 14 & 31.8 & & \\
\hline 13 & 28.3 & 28.3 & 28.3 \\
\hline 12 & 25.2 & & \\
\hline 11 & 22.4 & 22.4 & \\
\hline 10 & 20.0 & & 20.0 \\
\hline 9 & 17.8 & 17.8 & \\
\hline 8 & 15.9 & & \\
\hline 7 & 14.1 & 14.1 & 14.1 \\
\hline 6 & 12.6 & & \\
\hline 5 & 11.2 & 11.2 & \\
\hline 4 & 10.0 & & 10.0 \\
\hline 3 & 8.91 & 8.91 & \\
\hline 2 & 7.94 & & \\
\hline
\end{tabular}

\subsubsection{Principle of Operation of MASS}

The MASS sensor uses the time-of-flight technique to determine the mass of a solar wind ion with a high degree of accuracy (Hamilton et al., 1990; Gloeckler, 1990). The sensor consists of a spherical segment, electrostatic deflection system followed by a time-of-flight high mass resolution spectrometer.

Solar wind ions of kinetic energy $E$, mass $M$, and ionization state $Q$ enter the electrostatic deflection system (see Figure 6) which acts as an ultraviolet (UV) trap and an energy per charge $(E / Q)$ passband filter. Ions which exit the deflection 


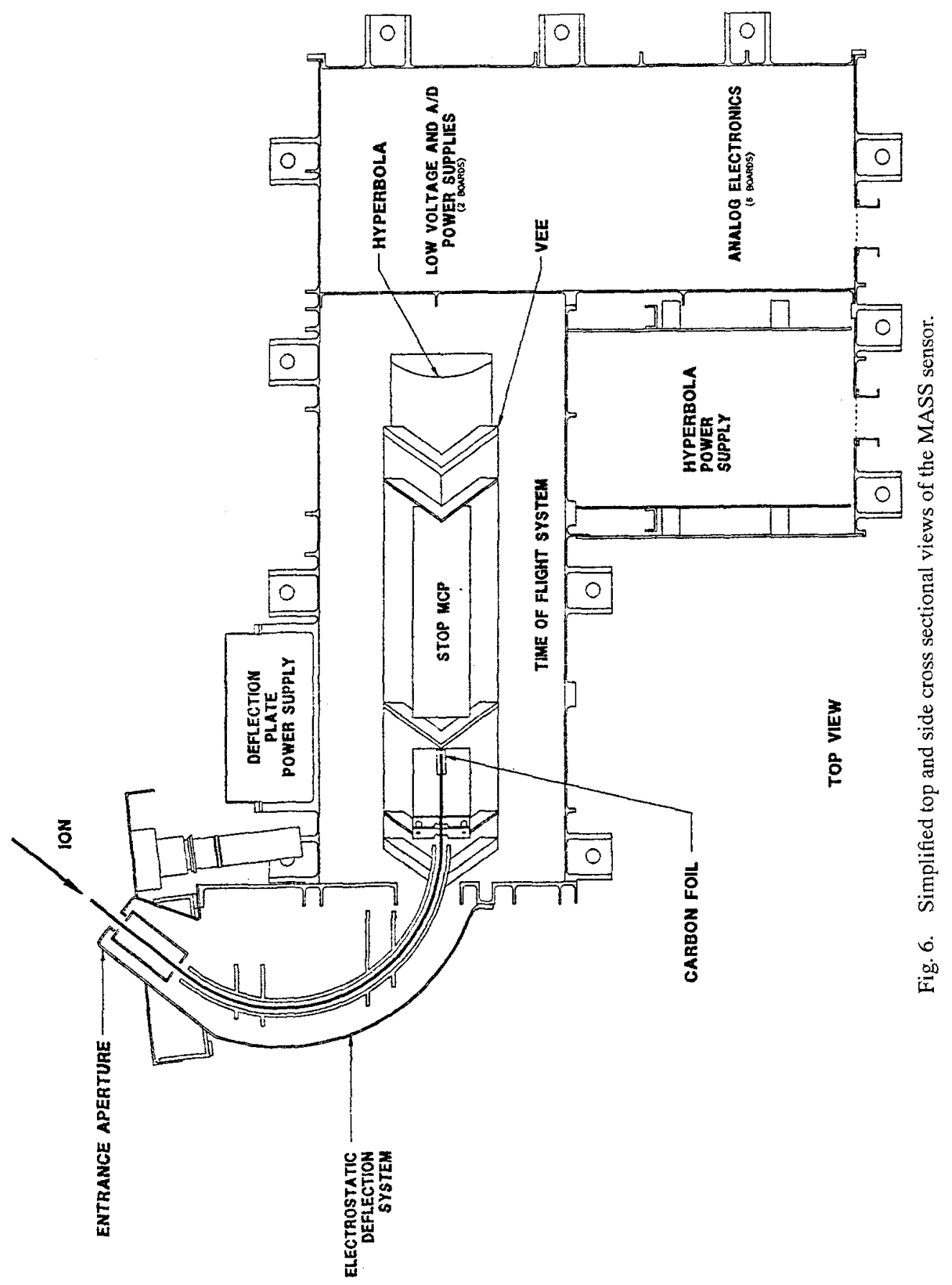




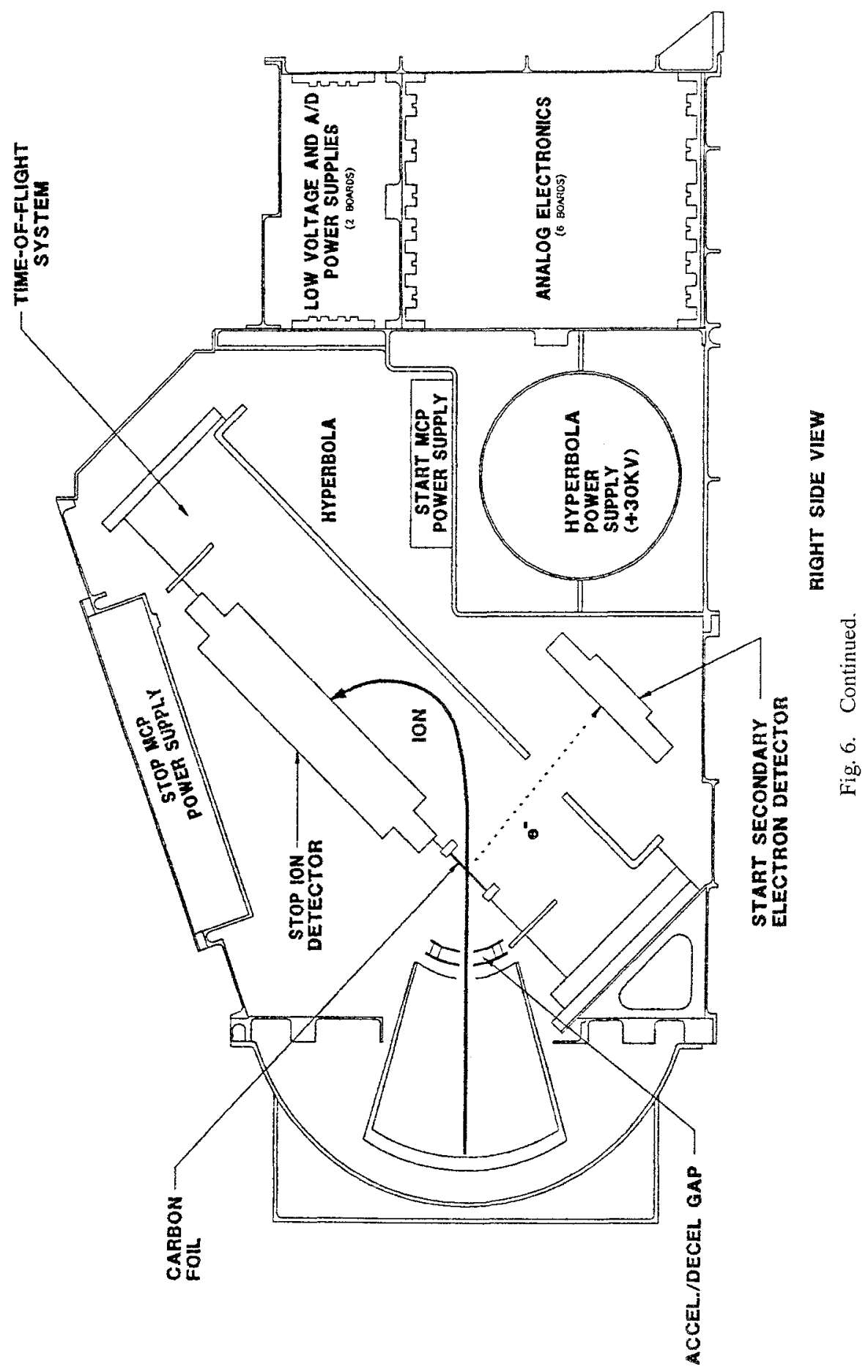


system have retained their original charge state and are confined to an energy per charge interval determined by the stepped deflection voltage.

Solar wind ions with an energy per charge $E / Q$ corresponding to the value set by the deflection system enter the mass analyzer section of the sensor after passing through a thin carbon foil. In passing through the carbon foil, the ions reach charge equilibrium and emerge predominantly as either neutrals or singly charged ions, with a smaller fraction having charges $Q^{*} \geq 2$ or -1 .

The carbon foil (located at $z=0$ ) also acts as the entrance to the TOF high mass resolution spectrometer. As the ion emerges from the foil, a small number (few to few tens) of secondary electrons are ejected from the foil. These electrons are accelerated to a microchannel plate assembly (MCP) and generate the start signal for the time-of-flight analysis. Positively charged ions are deflected back to a second, large area MCP assembly (also located at $z=0$ ), thereby generating the stop signal for the time-of-flight analysis. The principle of operation of the mass analyzer is to measure the time-of-flight $(\tau)$ of the predominantly singly ionized particles in a static retarding harmonic electric potential $\left(V \propto z^{2}\right.$, where $z$ is the vertical distance from the carbon foil). In such a potential $\tau$ is independent of the magnitude and angle of the velocity of the emerging ion and depends only on its $M / Q^{*}$ value:

$$
\tau \propto\left(M / Q^{*}\right)^{1 / 2} .
$$

Hence, a measurement of $\tau$ in this harmonic potential yields an unambiguous value of $M / Q^{*}$ and, for $Q^{*}=1$, of $M$. Typical times-of-flight range from 60 to $460 \mathrm{~ns}$. Since $\tau$ can easily be measured with the precision of a fraction of a nanosecond, the ion mass is determined to a high degree of accuracy $(M / \Delta M>$ $100)$.

\subsubsection{Description of the MASS Instrument}

3.3.2.1. MASS Deflection Analyzer Ions enter the MASS sensor through a simple entrance aperture. The deflection system has a spherical segment configuration ( $7.0 \mathrm{~cm}$ radius, $5.0 \mathrm{~mm}$ gap, $128^{\circ}$ deflection angle, and $\pm 20^{\circ}$ elevation angle). The black coated deflection plates prevent ultraviolet (UV) radiation from entering the time-of-flight analyzer. A single high voltage power supply steps the voltage on the inner (negative) deflection plate once per spin while the outer plate is held at ground. The voltage steps are logarithmically spaced from $-77 \mathrm{~V}(0.5 \mathrm{keV} / \mathrm{e})$ to $-1800 \mathrm{~V}(11.6 \mathrm{keV} / \mathrm{e})$ in $64 \mathrm{steps}$. The system has $\sim 5 \%$ energy/charge resolution.

3.3.2.2. MASS Time-of-FlightMass Analyzer Ions which exit the deflection system have retained their original charge state and are confined to a $5 \%$ energy/charge band. Ions pass through a $4 \times 15 \mathrm{~mm}$, grid-supported, $2 \mu \mathrm{g} \mathrm{cm}^{-2}$ carbon foil causing emission of secondary electrons which are rapidly accelerated through a hole in the hyperbola (see below) and reach the start chevron microchannel plate (MCP) assembly where their arrival time is recorded. Ions emerging from the start 


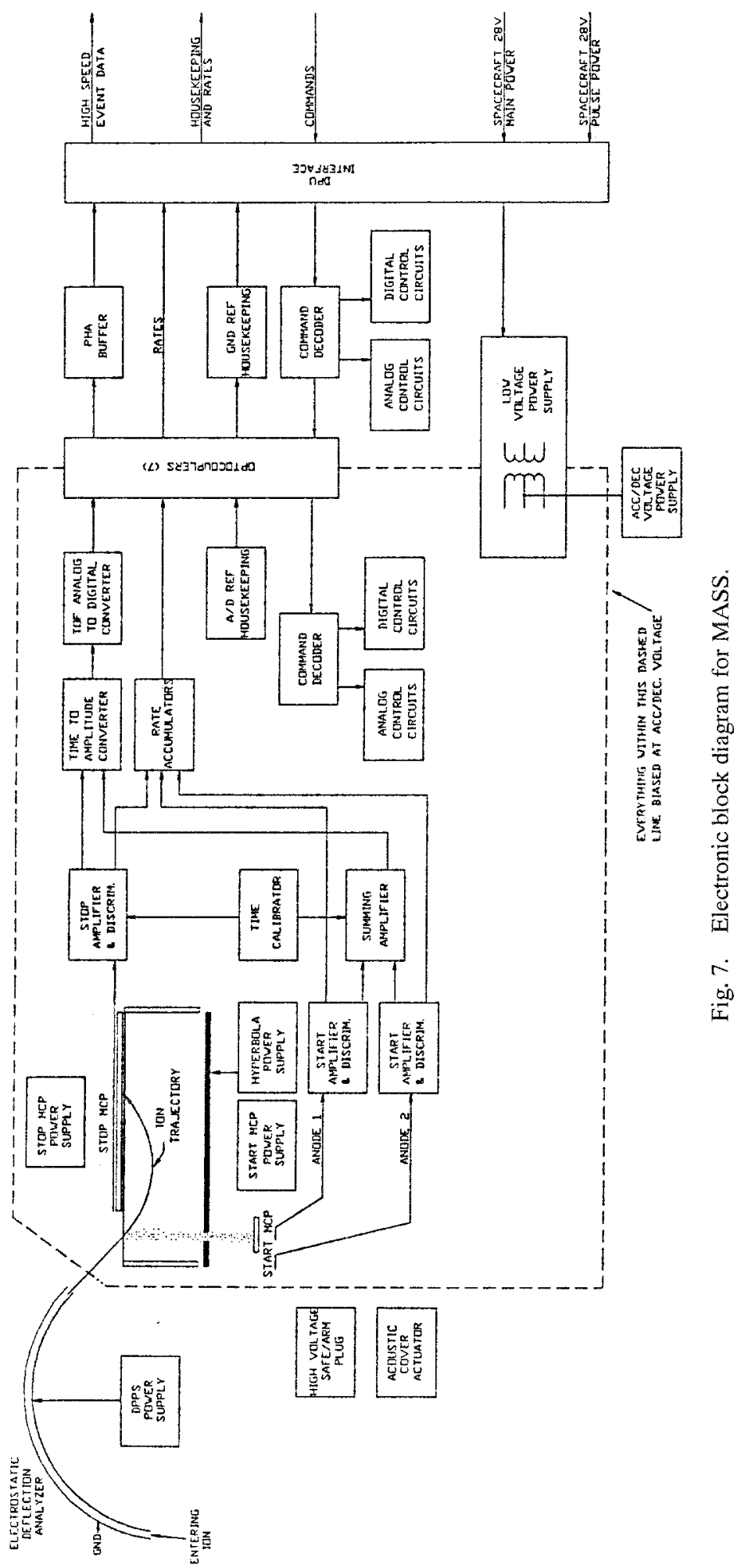


foil are most likely to have a 0 or +1 charge state with a much smaller fraction being +2 or (for some species) -1 . Neutrals and negatively charged ions will strike the high voltage plate (hyperbola) of the mass analyzer.

A static harmonic potential region is created in the mass analyzer system between the hyperbolically-shaped positive potential plate and a V-shaped plate at low potential. Positively charged ions emerging from the start foil will be deflected in this region and will hit the large area $(10 \times 1.5 \mathrm{~cm})$ stop chevron $\mathrm{MCP}$ assembly. Because of the harmonic potential inside the mass analyzer, the times of flight of positively charged ions depend only on their mass/charge and, since most have charge state +1 , only on their mass. The maximum voltage of the hyperbolic deflection plate is $+30 \mathrm{kV}$ (but adjustable downward).

\subsubsection{MASS Acceleration/Deceleration System The time-of-flight section and its associated electronics are floated at an adjustable acceleration/deceleration potential $V_{A / D}$. This potential is adjusted once per spin and may be set negative to accelerate low energy solar wind ions through the foil and thus increase the yield of +1 ions after the foil, or set positive to decelerate higher energy solar wind ions so that they are contained within the time-of-flight section. $V_{A / D}$ has 256 linearly-spaced values from -6.0 to $+1.7 \mathrm{kV}$.}

\subsubsection{MASS Modes of Operation In its normal mode of operation the MASS} deflection and acceleration/deceleration voltages will be stepped once per spin. The deflection analyzer has 64 settings $(0-63)$ with $5.1 \%$ separation between steps. The nominal sequence will comprise 60 steps starting with step $59(9.5 \mathrm{keV} / \mathrm{e})$ and ending with step $0(0.5 \mathrm{keV} / \mathrm{e})$. The acceleration/deceleration voltage will depend on the current step number and the speed of solar wind alpha particles measured during the previous cycle.

There will be an optional mode in which the stepping sequence reverses when a selected maximum Start MCP counting rate is reached. This mode can be used to avoid the solar wind protons and reduce fluence on the Start MCP. Another option is to set a given DPPS voltage for each of the 60 steps in the sequence and specify the value of $V_{A / D}$. This option will be used during calibration, for example.

\subsection{THE DATA PROCESSING UNIT (DPU)}

The DPU interfaces between the WIND spacecraft and the SWICS, MASS, and STICS sensors. It will handle all command processing, telemetry formatting, analog interfaces, power switching, alarm monitoring, and cover deployment mechanisms control for the instruments. Through the use of the spacecraft-provided timing and spin signals, the DPU will periodically collect TOF and energy pulse height data from the instruments via serial command and data lines. These data will be processed on board, sorted by category and type of event, and compressed 
into the available telemetry stream. The other principal functions of the DPU are described below.

\subsubsection{Functional Description of the DPU}

3.4.1.1. Fast Classification of Ions The DPU will execute fast classification of ions analyzed in each of the sensors according to the ion mass $(M)$ and mass per charge $(M / Q)$ for collection and storage of ion species rates. Fast look-up table techniques are used to establish a correspondence between the ENERGY $(E)$ and TIME-OF-FLIGHT $(T)$ pulse height data contained in the SWICS and STICS event words and the positions of the mass $(M)$ and mass-per-charge $(M / Q)$ surfaces in the $T$ vs $E$ parameter space. The classification algorithm used to generate the lookup tables is derived from the fundamental relations presented in Section 3.1.1 and includes several instrumental parameters, such as the energy-per-charge value of the deflection voltage step, the voltage across the post acceleration gap, the energy loss in the carbon foil, and the pulse height defect in the solid state detector. For the MASS sensor, the classification involves only the Time-of-Flight $(T)$ pulse height data and the hyperbola voltage. The $M$ and $M / Q$ values for SWICS and STICS and the $T$ or $M$ values for MASS returned by the fast classifier are used to increment appropriate storage registers corresponding to the Basic Rates, Matrix Rates and Matrix Elements.

Basic Rates. Basic Rates correspond to counting rates of ion species groups that fall into specified regions of $E-T$ space, defined by coarse resolution 'boxes' in $M$ vs $M / Q$ space for SWICS or STICS, or T space for MASS. These rates contain partial directional information. A Basic Rate is incremented by the DPU when the $M$ and $M / Q$ (or $T$ for MASS) calculated for an event word falls within the appropriate pulse height range. These rates are used for the normalization of the direct pulse height words (see section below on direct pulse height selection and storage).

Matrix Rates. Matrix Rates correspond to the counting rates of selected ion species defined by medium resolution rectangular 'boxes' in a logarithmic $M$ vs $M / Q$ space for SWICS and STICS, or $T(M)$ space for MASS. The boundaries of these rate boxes are defined in a DPU internal table which can be altered by RAM LOAD commands. A limited number (due to telemetry constraints) of these rates include directional information. For SWICS and STICS, the DPU uses the spacecraft spin clock and sun pulse to apportion the azimuthal (equatorial) plane into sixteen $22.5^{\circ}$ sectors. For some rates, this information is condensed by the DPU into eight $45^{\circ}$ equatorial sectors or into one omnidirectional sector. For STICS, the DPU distinguishes among six $26.5^{\circ}$ polar sectors covering $\pm 79^{\circ}$ out of the ecliptic plane by using the START anode identifier contained in the STICS pulse height event word. For some rates, this information is condensed by the DPU into three $53^{\circ}$ polar sectors or one omnidirectional sector. For the MASS sensor, the Matrix Rates are non-sectored. 
Matrix Elements. Matrix Elements in the SWICS telemetry correspond to fine resolution $M$ vs $M / Q$ rate bins covering a selected portion of the $M$ vs $M / Q$ parameter space. The bin size is on the order of the resolution of the sensor (typically $20 \%$ in mass and 3\% in mass/charge for SWICS). Because a large number of rate bins are involved, the matrix elements are accumulated over an entire instrument cycle and then are incrementally read out over the succeeding cycle. Information concerning the voltage step (hence $E / Q$ ) over which a particular event occurred is not retained.

\subsubsection{Direct Pulse Height Events The DPU will collect and store pulse} height data, determine event priority and execute appropriate event sequencing. The most detailed information about the energy (SWICS or STICS), time-of-flight, identification of triggered detector, priority range, and arrival direction (up to sixteen sectors) is contained in the direct pulse height analysis (PHA) words. The DPU collects and formats the required information for each PHA word, establishes the appropriate priority range category, and selects a subset of the available PHA words for transmission to the spacecraft telemetry. The priority range is defined by rather coarse ion species grouping in Mass vs Mass per Charge space (or $T$ space for MASS) and by sector groups. The species grouping (e.g., protons and helium; singly ionized heavy ions; and multiple-charged heavy ions) allows the transmission of a significant fraction of rare events (such as solar wind $\mathrm{C}, \mathrm{N}$, $\mathrm{O}, \mathrm{Fe}$, and interstellar pickup ions), while not completely excluding the more abundant ion species (e.g., helium). The sector grouping allows a sampling of events over all pitch angles (STICS) or over the solar/non-solar sectors (SWICS and MASS). Priority range selection affects only the direct pulse height data and the corresponding Basic Rates.

3.4.1.3. Data Compression and Formatting The DPU will compress the contents of each counting rate register into an 8-bit floating point representation, and format all data and transfer this information to the spacecraft. All sensor-generated rates and the DPU-generated species rates (derived from the pulse height event words) are compressed using either a uniform floating point code (maximum count accumulation of $5 \times 10^{5}$ ) or a non-uniform floating point code (maximum count accumulation of $7 \times 10^{6}$ ). The rate compression code is selected by command.

3.4.1.4. Control of Sensors The DPU will perform all necessary control functions for the instruments. It accepts and executes ground commands, monitors the experiment status (and initiates emergency procedures when appropriate), and, on command, executes an on-board calibration sequence of the analog electronics.

\subsubsection{Description of the DPU}

The electronic block diagram of the DPU is shown in Figure 8. The DPU can be subdivided into five functional parts:

(1) Interfaces to each of the three instruments. 


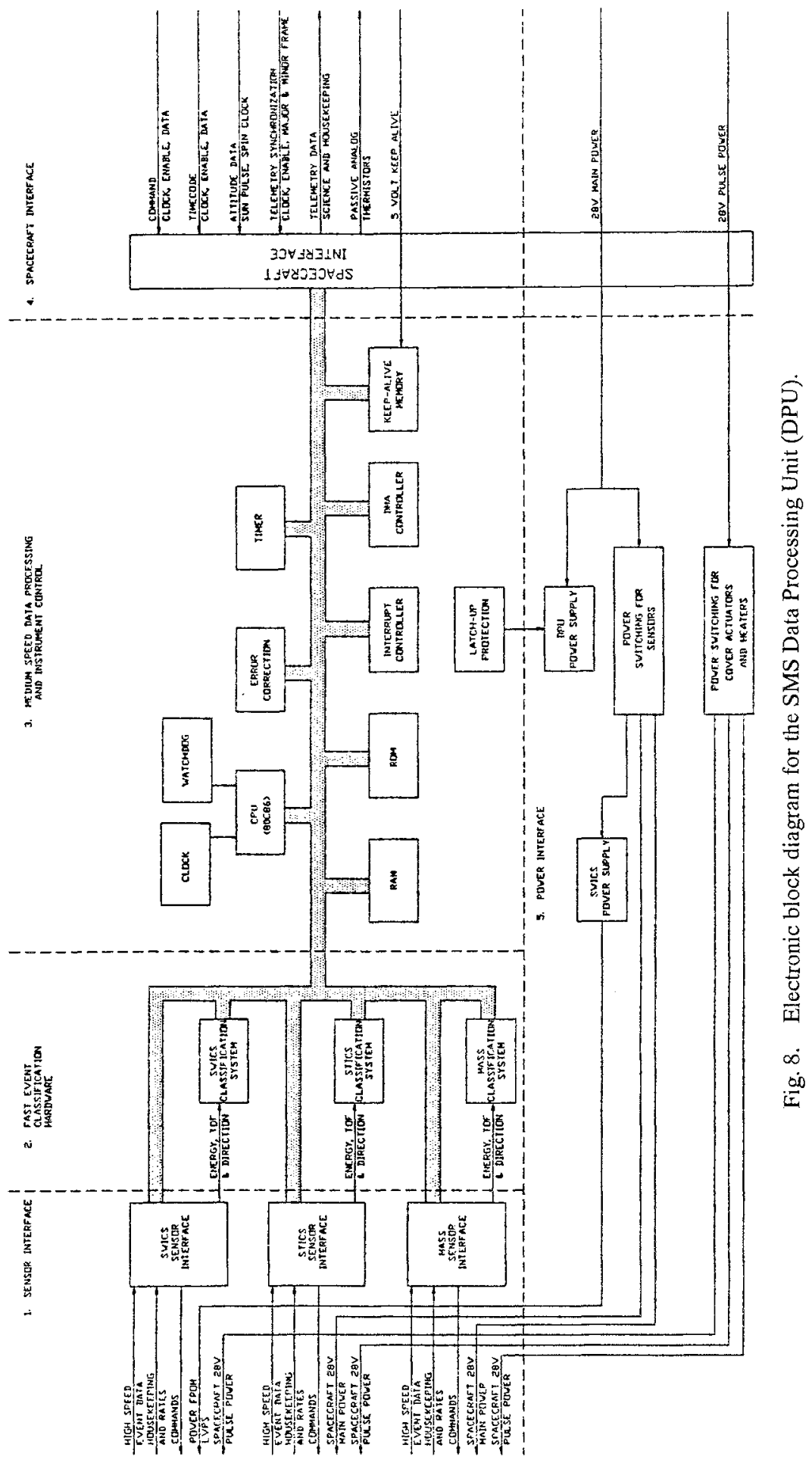




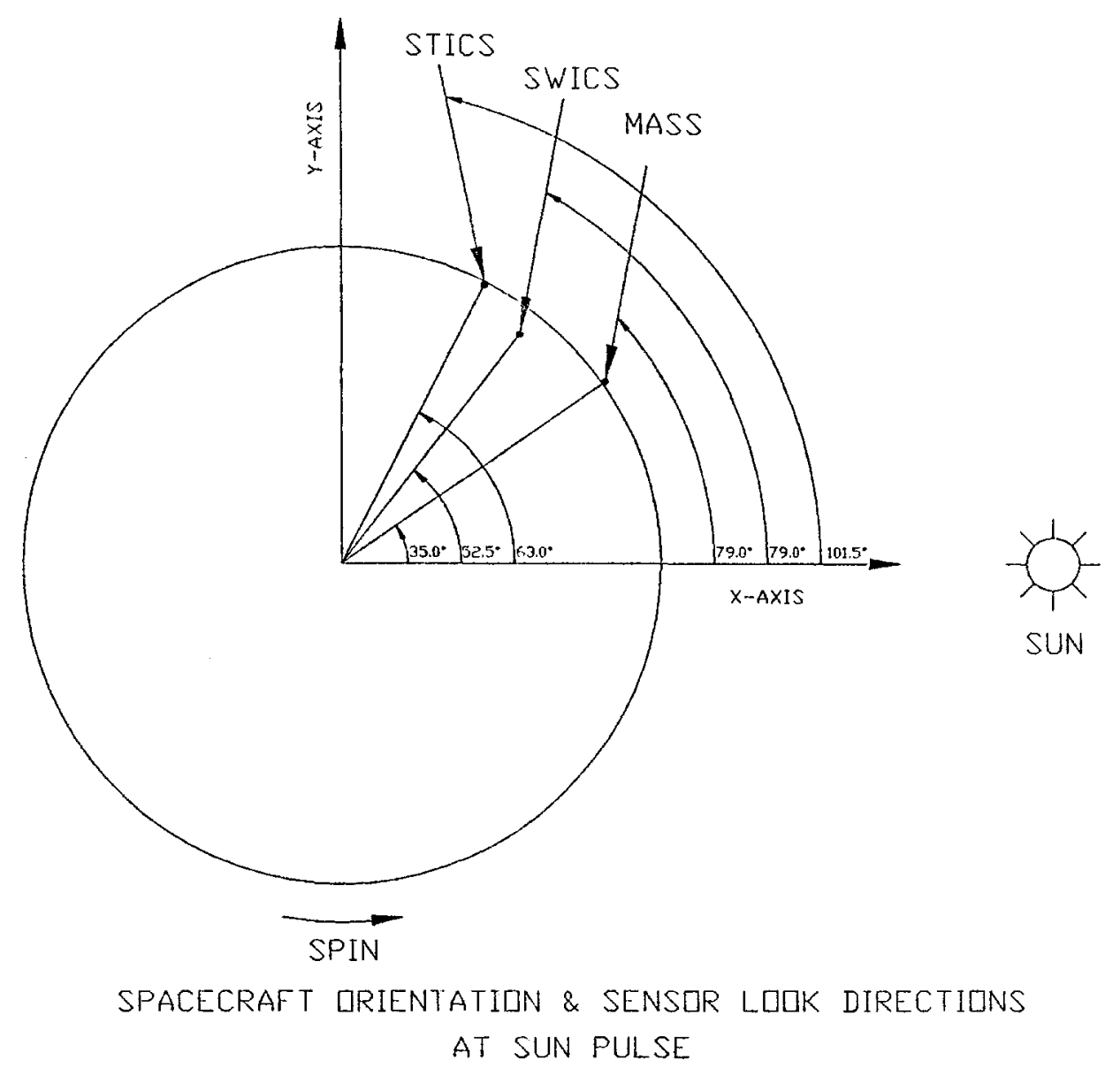

Fig. 9a. Field-of-view pointing directions for STICS, MASS, and SWICS relative to the WIND spacecraft $x$-axis.

(2) Fast data processing by means of task-dedicated hardware.

(3) Medium speed data processing and instrument control by means of a $80 \mathrm{C} 86$ microprocessor system.

(4) Interface to the WIND spacecraft.

(5) Low voltage power converters for the DPU and SWICS, and power control for the entire SMS experiment.

Data flow is primarily from part (1) to part (4), while commands flow the other way. The data rate is reduced from a maximum of $4 \mathrm{Mbits} \mathrm{s}^{-1}$ at the entrance of part (1), i.e., the sensor outputs, to the experiment telemetry rate of 870 bits s $^{-1}$ at the output of part (4). Much of this data compression and processing is performed by the dedicated hardware in part (2). 
The high-speed classification will be implemented through the use of direct RAM-based table looked-up techniques. This approach, made feasible by new highdensity RAMs, makes it possible for the mass and mass per charge classification algorithms to be changed in flight if necessary. The contents of the tables will be calculated on board, based upon a limited number of uploaded parameters.

All instrument power switching is handled in the DPU via MOSFETs. This will support power cycling of instrument subsystems (preamps, event processors, etc.), as well as basic instrument control. Other special requirements, such as RAM loads (for program updates or parameter loads) will also be supported.

Because of the limited access to real-time ground-based control, the instrument will also incorporate a sophisticated housekeeping monitor. The instrument operating system will continuously cycle through a number of instrument housekeeping parameters, such as temperatures, voltages, currents, and status. If these parameters are found to be outside predetermined limits, the DPU will initiate an appropriate alarm routine, and set an alarm bit in the real-time instrument status.

The DPU consists of a stack of 12 multi-layer boards $(156 \times 170 \mathrm{~mm})$ interconnected by a common multi-layer mother board. Two of the boards are the DC/DC-Converter and the Power Control Board.

\section{Instrument Characteristics and Requirements}

\subsection{INSTRUMENT POINTING DIRECTIONS AND FIELDS OF VIEW}

The center pointing direction of each of the three instrument's fan-shaped field of view is perpendicular to the spacecraft spin axis (i.e., in the ecliptic plane), with that of STICS offset by $22.5^{\circ}$ from those of SWICS and MASS which point in the same direction as shown in Figure 9(a). The wide acceptance angles $\left( \pm 20^{\circ}\right.$ for MASS, $\pm 22.5^{\circ}$ for SWICS and $\pm 80^{\circ}$ for STICS) combined with sectoring (sixteen equal $22.5^{\circ}$ sectors) provides directional information both in and off the ecliptic plane. In particular, the $\pm 80^{\circ}$ acceptance angle of the STICS instrument permit 3-dimensional anisotropy measurements.

\subsection{MASS, SIZE, AND POWER}

The SMS experiment mass and power requirements are $27.28 \mathrm{~kg}$ and $19.9 \mathrm{~W}$, respectively. The mass allocation is exclusive of the mass of the spacecraft harnesses connecting the DPU to the three sensors and to the spacecraft. The mass, power requirement and the approximate size of each of the five units are given in Table III.

\subsection{TELEMETRY}

Spacecraft telemetry is divided into Major Frames. Each Major Frame consists of 250 subframes which are in turn composed of 256 bytes. The SMS experiment has 

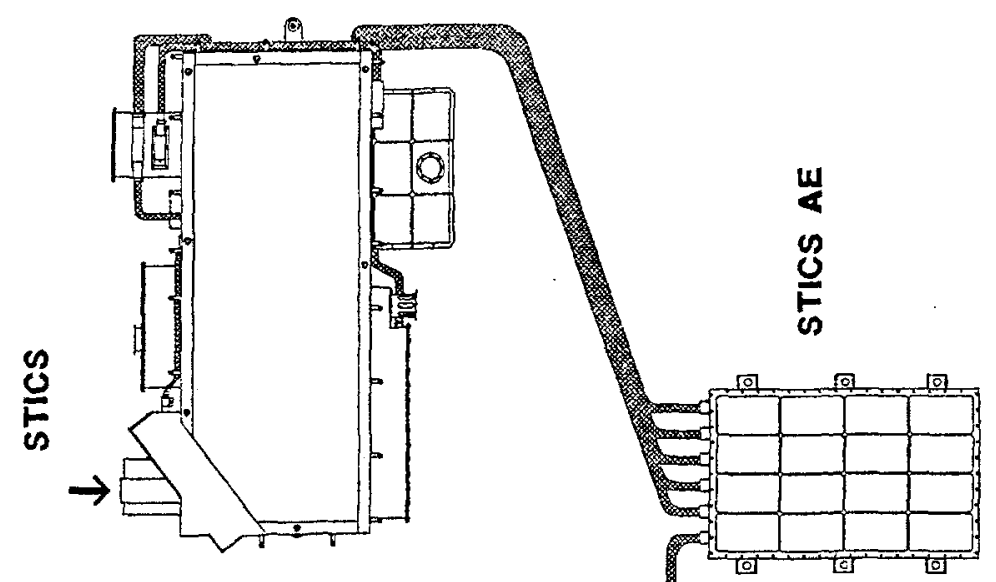
TABLE III

Power, mass and size for each of the five SMS experiment units

\begin{tabular}{lccc}
\hline Unit name & Power (W) & Mass $(\mathrm{kg})$ & \multicolumn{2}{c}{ Size $(\mathrm{mm} \times \mathrm{mm} \times \mathrm{mm})$} \\
& & & $\mathrm{l}$ w \\
\hline DPU & 4.0 & 5.26 & $200 \times 192 \times 190$ \\
SWICS sensor & 4.2 & 6.50 & $415 \times 432 \times 290$ \\
MASS sensor & 4.7 & 5.90 & $452 \times 362 \times 259$ \\
STICS analog elec & 4.3 & 2.32 & $229 \times 165 \times 132$ \\
STICS sensor & 2.7 & 7.30 & $435 \times 290 \times 359$ \\
Total SMS expmt. & 19.9 & 27.28 & \\
\hline
\end{tabular}

been allocated 40 bytes of science data per sub-frame. In the normal spacecraft telemetry mode this amounts to 869.53 bits s $^{-1}$.

All SMS science measurements and telemetry are spin-related. During one spin the deflection voltage ( $E / Q$ step) is held constant. Deflection voltage transitions are synchronized with respect to the spacecraft sun pulse (transitions for the three sensors have individually commandable time offsets). The normal deflection voltage sequence covers 60 steps for SWICS and MASS, and 10 steps for STICS.

Science data, gathered during one spin period, forms an Experiment Data Block (EDB). The amount of data in an EDB depends on the spacecraft spin rate and telemetry mode, but the average time duration of an EDB has to equal the average spin period. An EDB contains an integer number of sub-frames and is allowed to start only on sub-frame boundaries. Each EDB contains a common header, a fixed amount of core data per sensor, and a variable amount of PHA information per sensor. In the normal science telemetry mode at a spin period of $3 \mathrm{~s}$, the EDB will be either 350 or 400 bytes, with an average of 364 bytes per EDB. The EDB adapts to the number of sensors that are active.

The EDB Layout can be divided into three parts:

(1) Common: there are eleven bytes of common data. These include a spin counter, and information about the telemetry mode, the number of subframes per $\mathrm{EDB}$, and the data allocation for each sensor.

(2) Core: when the spacecraft is in the normal science telemetry rate there are 62 bytes of SWICS core data, 27 bytes of MASS core data, and 90 bytes of STICS core data. These data include basic rates, matrix rates, matrix elements, deflection voltage step number, and essential engineering rates.

(3) PHA: the amount of PHA data varies with the number of subframes per EDB. The nominal allocation is 10 PHA events ( 4 bytes each) for SWICS, 23 PHA events ( 2 bytes each) for MASS, and 22 PHA events ( 4 bytes each) for STICS. If one of the sensors is off or if the number of PHAs from one sensor is less than its nominal allocation, the unused date area is released to the other two sensors. 
The experiment data collection cycle is based on the maximum number of steps in a deflection voltage cycle. A Science Record consists of 60 EDBs and a Housekeeping Data Block of 280 bytes and is formed from the instrument status engineering data.

In addition to the science data, there are 75 bytes of housekeeping data in each spacecraft Major Frame. Five bytes are passive analog temperatures measured by the spacecraft. When the instrument is on, seventy bytes of serial digital housekeeping are used to monitor instrument health.

\subsection{THERMAL}

Thermal design requirements for the SWICS and STICS sensors are for in-orbit operating temperatures of 5 to $-20^{\circ} \mathrm{C}$. These thermal requirements are driven by solid-state detector operating and survival temperature limits, and are achieved by thermally isolating the SWICS and STICS sensors from the spacecraft and through use of thermal blankets, radiators and heaters on these units. The other three units, including the MASS sensor, do not contain solid-state detectors and thus require no special thermal control. These units are thermally coupled to the spacecraft.

\section{Configuration}

The SMS experiment is comprised of 5 units: the SWICS sensor, the MASS sensor, the STICS sensor, the STICS Analog Electronics box, and the Data Processing Unit. A schematic of these assemblies and of the interconnecting harness is shown in Figure 9(b).

The three sensors shown on top of Figure 9(b) (but not in their actual orientation on the WIND spacecraft, see Figure 9(a)) detect and measure the characteristics of ion populations in space. These ions enter each of the sensors in the directions shown by arrows through an entrance system protected prior to and during launch by acoustic covers which are opened after launch by paraffin motor actuators. The entrance system for each sensor protrudes through a fairing plate which in turn is part of the spacecraft outer surface. The rest of the sensors as well as the DPU and STICS analog electronics are inside the spacecraft, mounted to one of the two spacecraft decks. The SWICS and STICS sensors are thermally isolated from the spacecraft, are enclosed within thermal blankets and have radiator surfaces as well as heaters for thermal control.

The sensors contain a combination of microchannel plates and solid state detectors, high voltage supplies ranging from a few hundred volts to $30 \mathrm{kV}$ and preamplifiers. SWICS and MASS also contain all the analog signal processing electronics as well as digital interface circuits to the DPU. For STICS these electronics are housed in a separate Analog Electronics box connected to the STICS sensor by means of a harness that includes shielded coaxial cables for analog signals. Digital data from 
the SWICS and MASS sensors and the STICS Analog Electronics box are sent to the DPU for mass and mass/charge classification, data storage, compression and formatting.

\section{Modes of Operation}

While numerous in-flight adjustments to the operations of the three instruments and the DPU are possible, it is planned use only one mode of operation for extended lengths of time. After initial turn-on and the ramp-up of high voltages, the performance of each sensor will be optimized to obtain the full range of spectral, species and angular coverage with reasonable time resolution. Only under especially compelling circumstances will we change in a significant way the mode of operation of any of the instruments.

\section{Key Parameter Data}

The SMS investigation will provide selected supra-thermal particle fluxes and solar wind parameter for several heavy ion species averaged over 6 min (or multiples of $6 \mathrm{~min}$ ). While the details have as yet not been determined, it is likely that the following data will be included.

(1) Solar wind $\mathrm{He}^{++}$pseudo-density, speed and kinetic temperature.

(2) Omni-directional fluxes of supra-thermal $\mathrm{H}^{+}$and $\mathrm{He}^{++}$from about 10 to $230 \mathrm{keV} / \mathrm{e}$ in 10 logarithmically spaced energy bands, and

(3) Angular distributions of 40 and $230 \mathrm{keV} \mathrm{H} \mathrm{H}^{+}$fluxes averaged separately over the 'north' and 'south' polar sectors.

\section{Instrument Calibration}

The SWICS and STICS instruments on the WIND spacecraft are virtually identical to the corresponding instruments flown on Ulysses, launched in 1990, and Geotail launched in 1992. In fact, the WIND/STICS sensor is an exact copy of the Geotail/STICS. There is, therefore, ample information on the flight performance and preflight calibrations for these two instruments (e.g. Gloeckler et al., 1992, 1983; Williams et al., 1991; Christon et al., 1994). MASS is a new design and has not been flown previously. Pre-flight calibrations of all three flight instruments with the flight DPU and Ground Support Equipment were performed in NovemberDecember 1993 at the Accelerator Facility at the University of Bern. The STICS flight unit was also calibrated at the Goddard Space Flight Center Accelerator. We measured the energy dependence of efficiencies for various ion species as a function of MCP bias level, geometrical factors (listed in Table I, as well as the angular and energy responses of electrostatic deflection systems over the entire 
field-of-view of each sensor. The DPU classification and its other functions were also verified. The Bern Facility was well suited for the calibration of especially the Solar Wind instruments SWICS and MASS. It was possible to obtain uniform (over areas of 30 to $50 \mathrm{~cm}^{2}$ ) and stable (in both energy and intensity) beams of ion species (e.g., $\mathrm{H}^{+}, \mathrm{He}^{+}, \mathrm{He}^{++}, \mathrm{O}^{+}, \mathrm{C}^{+}, \mathrm{N}^{+}, \mathrm{Ne}^{+3}, \mathrm{Ar}^{+3}, \mathrm{Kr}^{+4}$ ) from a few hundred $\mathrm{eV} / \mathrm{e}$ to about $60 \mathrm{keV} / \mathrm{e}$. The Goddard Facility was used in order to calibrate the high energy range ( $>60 \mathrm{keV} / \mathrm{e}$ ) of STICS. The amount of calibration data obtained was far too large to be shown here in its entirety. Instead we give just a few examples of relevant calibration or post-launch performance data for each of the three instruments.

\subsection{SWICS EFFICIENCIES}

Knowledge of the efficiencies of the MCPs as a function of energy of the various elements is required to obtain fluxes and relative abundances of solar wind ions. The stop efficiencies of SWICS for $\mathrm{He}, \mathrm{C}, \mathrm{O}, \mathrm{Ar}$, and $\mathrm{Kr}$ as a function of energy from 1 to $10 \mathrm{keV} \mathrm{amu}{ }^{-1}$ are shown in Figure 10. Start efficiencies have a similar energy and species dependence. In SWICS the relevant energy is the ion energy after post-acceleration. For example pickup $\mathrm{O}+$ ions at twice the solar wind speed would be post-accelerated to $2 \mathrm{keV} \mathrm{amu}{ }^{-1}$ at $28 \mathrm{kV}$ post-acceleration voltage and would have a stop efficiency of 0.3 .

\subsection{STICS THREE-DIMENSIONAL ANGULAR RESPONSE}

The distinguishing feature of STICS is its full 3-dimensional angular response, which along with its large geometrical factor, its high (compared to SWICS and MASS) upper energy limit and its mass and mass/charge resolution capabilities makes STICS well suited for measuring the full distribution functions of suprathermal ions of various origins. The WIND/STICS is identical to the Geotail/STICS which has been operating nominally since launch. The Geotail/STICS angular spectra are shown in Figure 11 for two different periods (labeled ' $a$ ' and ' $b$ ') of plasma sheet incursions. The polar angle is plotted against the azimuthal (ecliptic plane) angle, with the azimuthal angle wrapped around by $90^{\circ}$ to provide continuity. The STICS sensor covers $360^{\circ}$ in the azimuthal direction in up to sixteen $22.5^{\circ}$ sectors, and $156^{\circ}$ in polar direction in up to six equal angular sectors. Relative particle intensities (coded by a linear color scale) for nominally ionospheric ions (a combined rate consisting of the sum of $\mathrm{O}^{+}, \mathrm{N}^{+}$, and molecular ions) are plotted in the top two panels, and a combined rate of solar wind $\left(\mathrm{He}^{++}\right)$and possibly mixed origin $\left(\mathrm{H}^{+}\right)$are shown in the bottom two panels. The particle fluxes have been integrated over the entire energy range of Geotail/STICS $(10-210 \mathrm{keV} / \mathrm{e})$. Period (a) indicates southward flowing ions, with the solar wind origin $\mathrm{H}^{+}$and $\mathrm{He}^{++}$ flowing primarily dawnward, and the ionospheric origin ions showing streaming in both the tailward and nearly earthward directions. Period (b) shows a beamlike tailward flow in the ecliptic plane for both types of ions. 


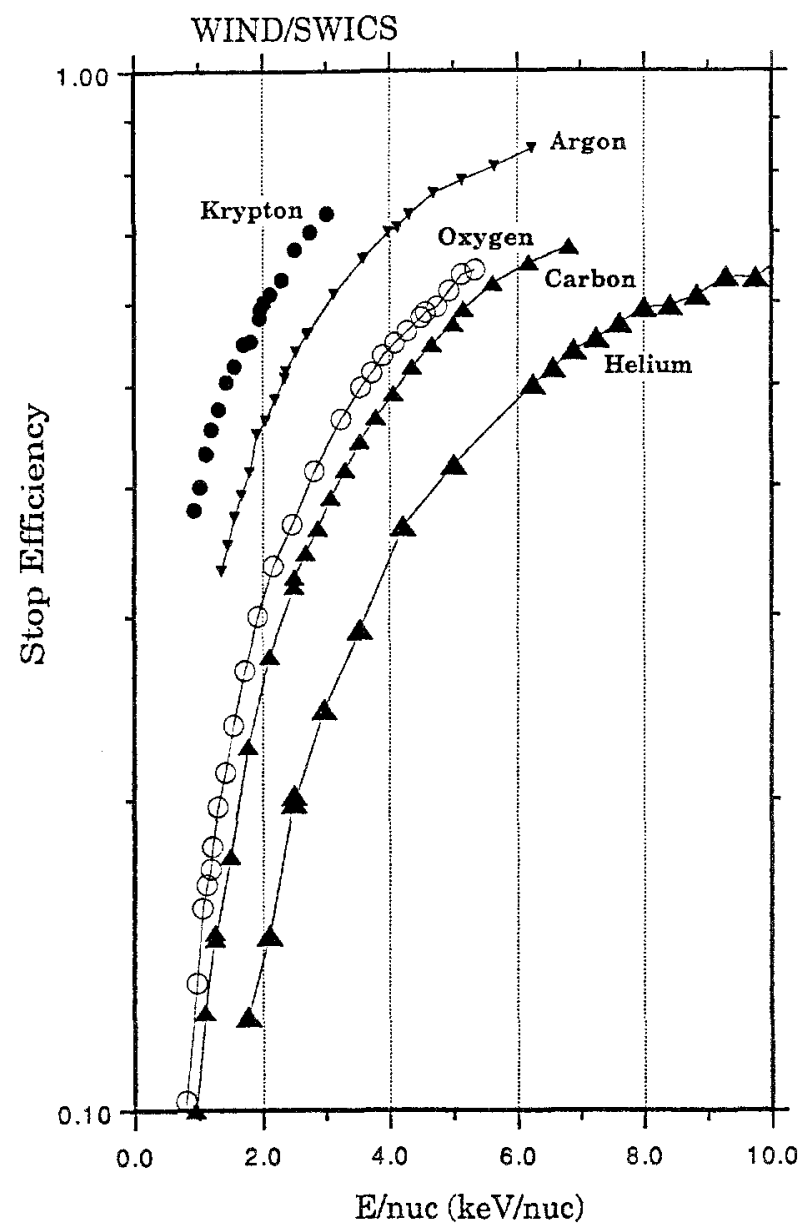

Fig. 10. Measured dependence of the WIND/SWICS stop efficiency on energy per nucleon for the indicated elements. The stop efficiency is the ratio of the Double Coincidence Rate to the Start Rate and reflects the effects of ion scattering caused by the carbon foil, the efficiency of generating back-scattered secondary electrons from the solid state detectors and adjacent surfaces, the efficiency of focusing these electrons onto the stop microchannel plate (MCP), and the efficiency of detecting these electrons by the stop MCP.

\subsection{MASS ELEMENTAL RESOLUTION}

The unique strength of the MASS instrument is its high mass resolution, making it possible to resolve for the first time all the solar wind elements up to and including $\mathrm{Ni}$, and many of their isotopes. To illustrate the mass resolution of MASS we show in Figure 12 results from the instrument calibrations at the University of Bern in November 1993. Data have been combined from four separate runs $\left(25 \mathrm{keV} \mathrm{C}^{+}\right.$, $\mathrm{N}^{+}$, and $\mathrm{O}^{+}$, and $15 \mathrm{keV} / \mathrm{e} \mathrm{Ne}{ }^{++}$). Isotopes such as ${ }^{13} \mathrm{C}$ and ${ }^{22} \mathrm{Ne}$ are not seen in this figure because the Bern Accelerator is very selective and passes only one 


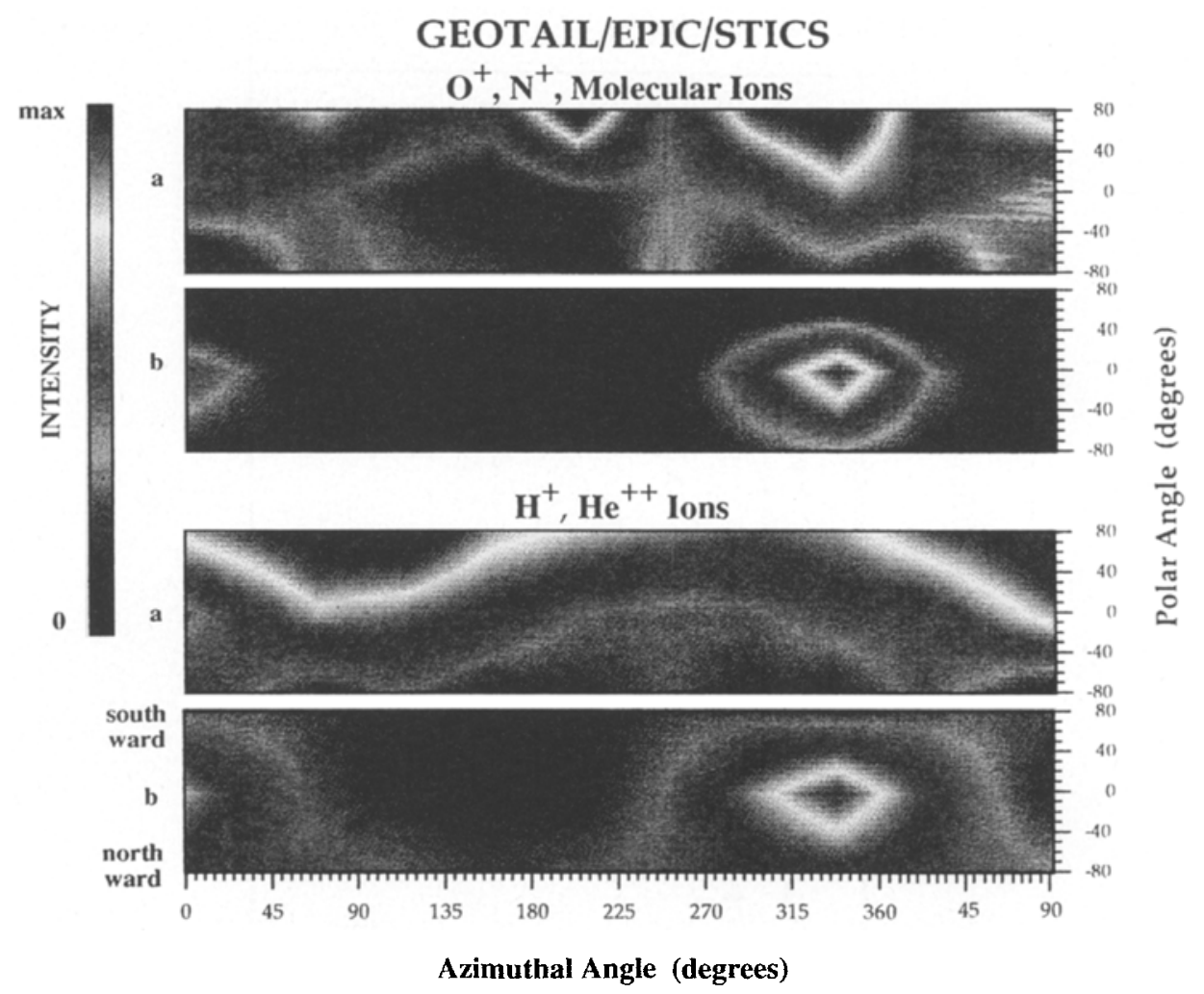

Fig. 11. Angular spectra for two different periods (labeled ' $a$ ' and ' $b$ ') measured by the Geotail/STICS in the distant magnetotail of the Earth. The identical STICS sensors on Geotail and WIND view $360^{\circ}$ in azimuthal direction in up to sixteen equal sectors and $156^{\circ}$ in polar direction in up to six equal sectors. As demonstrated in this figure, the close to $4 \pi$ angular coverage is essential in order to observe the highly anisotropic distributions of supra-thermal ions. (Based on Figure 3 of Christon et al., 1994; see text for explanation).

isotope at a time. The channel number is proportional to the time-of-flight $(\tau)$ with channel 4095 corresponding to $515 \mathrm{~ns}$ and the mass $M=(\tau / 59)^{2}$, where $\tau$ is in ns and $M$ in amu. It is evident from these data that MASS flight unit has achieved a mass resolution $M / \Delta M$ (FWHM) of over 100 , sufficient to resolve many of the isotopes in the solar wind.

\section{Summary}

The Solar Wind and Suprathermal Ion Composition Investigation on the WIND spacecraft will obtain detailed information on the thermal and suprathermal ion populations in the solar wind upstream of the Earth's magnetosphere and for brief periods inside the magnetosphere, with an emphasis on compositions of these plasmas. This information is required for studies of a variety of solar, heliospheric and 


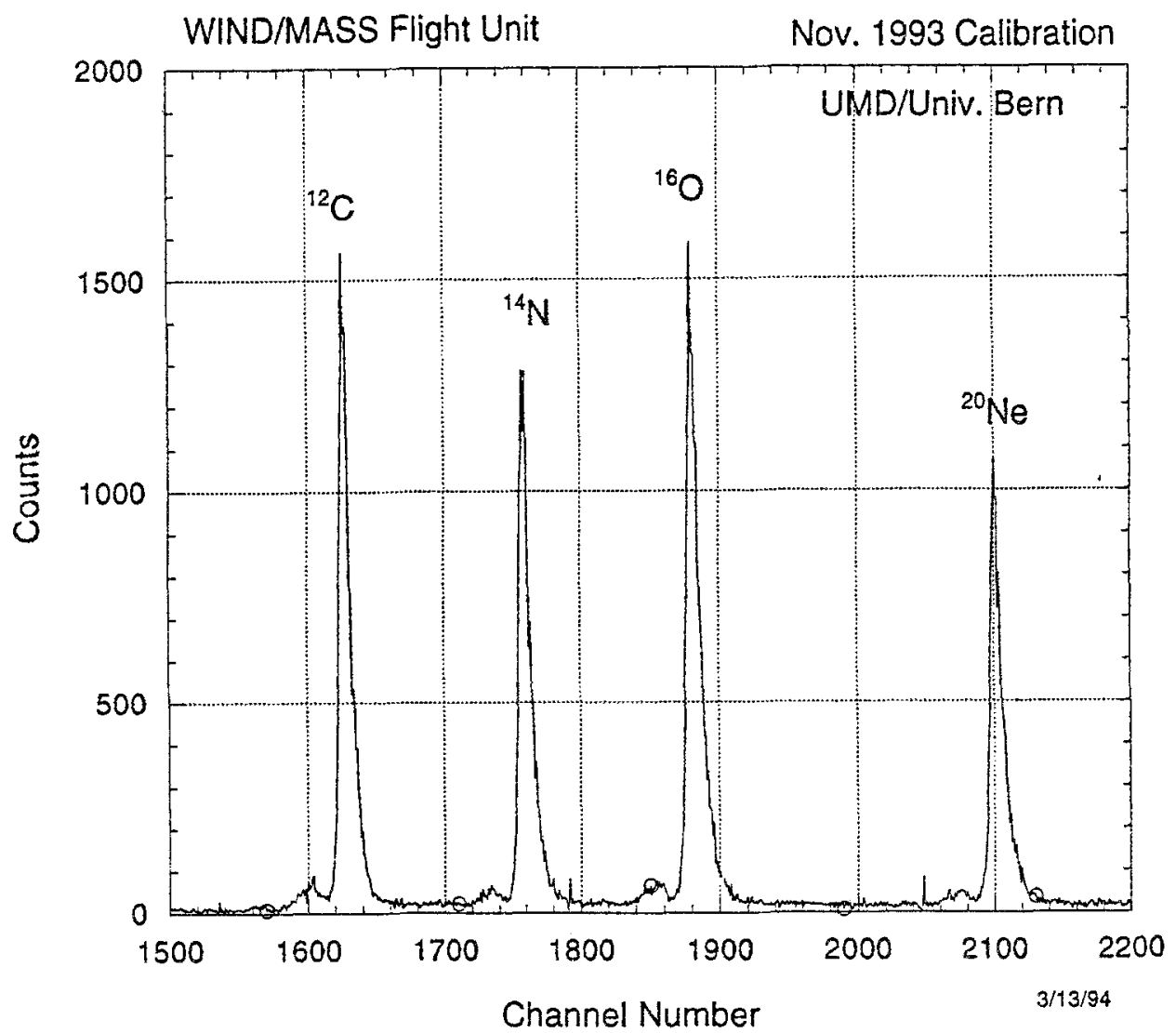

Fig. 12. Time-of-flight histograms, dispiaying the mass resolution of a the MASS sensor, obtained during the final calibration of the flight unit at the University of Bern Calibration Facility. Data have been combined from four separate runs: $25 \mathrm{keV} \mathrm{C}^{+}, \mathrm{N}^{+}$, and $\mathrm{O}^{+}$, and $15 \mathrm{keV} / \mathrm{e} \mathrm{Ne}{ }^{++}$. In each case the hyperbola potential was $23.0 \mathrm{kV}$ and the acceleration/deceleration potential was zero.

magnetospheric (bow shock) phenomena, and is important for interpreting results from other GGS and ISTP spacecraft, heliospheric missions such as Ulysses, and various space and ground based observations of the solar photosphere and lower corona. These data, especially in combination with measurements from other instruments on WIND, other GGS and ISTP spacecraft and Ulysses, as well as ground and space observations of the sun, will make possible a number of new studies of solar, heliospheric and magnetospheric phenomena. These scientific objectives require measurements provided by three complementary instruments. The Solar Wind Ion Composition Spectrometer (SWICS) will determine routinely the charge states and differential energy fluxes of the most abundant solar wind heavy ions $(\mathrm{H}, \mathrm{He}, \mathrm{C}, \mathrm{O}, \mathrm{Ne}, \mathrm{Mg}, \mathrm{Si}$, and $\mathrm{Fe}$ ) from 0.5 to $30 \mathrm{keV} / \mathrm{e}$ ). The Supra-Thermal Ion Composition Spectrometer (STICS) complements SWICS by measuring the supra-thermal plasma primarily above $30 \mathrm{keV} / \mathrm{e}$ (the upper range of SWICS). The 
large (compared to SWICS) geometrical factor and the full 3-dimensional viewing capabilities of STICS are necessary for measuring the low fluxes and complicated angular distributions of suprathermal ion populations. Finally, the High Resolution Mass Spectrometer (MASS) will measure for the first time the elemental and isotopic composition of the solar wind plasma with far greater precision than is possible with SWICS (i.e., elements $\mathrm{Na}, \mathrm{Al}, \mathrm{S}, \mathrm{Ar}, \mathrm{Ca}, \mathrm{Cr}$, and $\mathrm{Ni}$, and isotopes of $\mathrm{Ne}, \mathrm{Mg}, \mathrm{Si}, \mathrm{S}, \mathrm{Ar}, \mathrm{Ca}$, and $\mathrm{Fe}$ ).

\section{Acknowledgements}

The development of the SMS experiment was an international effort involving the Space Physics group in the Department of Physics at the University of Maryland, the Physikalisches Institut, Universität Bern, Bern, Switzerland, the Institut für Datenverarbeitungsanlagen, Universität Braunschweig, Braunschweig, Germany, and the Max-Planck-Institut für Aeronomie, Katlenburg-Lindau, Germany. Many individuals at these institutions contributed greatly in the design, development, fabrication testing and calibration of the three SMS instruments and the DPU. We are particularly grateful to the following individuals. At the University of Maryland to Ed Tums for the electrical engineering and testing of the analog electronics and the high voltage systems of all instruments including the $30 \mathrm{kV}$ supplies for SWICS and MASS, and the integration of the complete STICS instrument; to Fred Wire for the design and testing of digital subsystems for all instruments, and the integration of the complete SWICS instrument; to Richard Pappalardo for the developing and testing the power systems for STICS and MASS, and the integration of the complete MASS sensor; to John Cain, Bob Cates and Jerry Rossano for the mechanical engineering, fabrication and testing of the STICS instrument; and to Paul Wilson and Mike Lungociu for the mechanical design of SWICS and MASS respectively; to Maurice Pairel and Hank Wats who did an excellent job laying out and assembling the many electronics boards for the instruments; to Mike Bullinger for fabricating and assembling the complicated TOF vs E telescopes of STICS; to Scott Lasley for his laboratory testing of sensor detector systems, especially for STICS, and for his invaluable support during the integration, testing and calibrations of the SMS instruments; to Peter Bedini and Herb Broer for developing and testing the GSE software; to Mike Collier, Wayne Shanks and Sham Chotoo for their help during instrument testing and calibration; to Pat Ipavich and Aileen DeSrosier for the financial management and reporting for the SMS experiment; to our secretary Cassie Jones for administrative help; and to George Shettle for parts procurement, instrument handling and shipping. At the University of Bern to Joe Fischer and Kurt Bratschi, who developed, fabricated and tested the deflection systems for MASS and SWICS, including its entrance collimator and deployable cover; to Roland Bodmer, Marc Gonin, Urs Mall, and Thomas Zurbuchen for their assistance in the ion-optical design, testing and calibration of the entrance systems of MASS 
and SWICS; and to Urs Schwab and Martin Steinacher for the operation of the Bern Calibration Facility during the many 24-hr days of calibrations of the SMS instruments. At the Technical University of Braunschweig to Dirk Lohse for the electrical design and testing of the DPU hardware and to Bernd Gerlach who wrote and debugged the extensive DPU software. At the Max-Planck-Institut für Aeronomie to Peter Winterhoff for checking out the analog electronics boards for SWICS. We thank Steve Brown and Claude Smith at Goddard for their help during calibrations of the MASS and STICS instruments and subsystems, and we appreciate the support of the GGS Project Office at the Goddard Space Flight Center, headed by John Hrastar, throughout the long years of development of the SMS experiment, and are particularly grateful to Ed Werner who helped us in many ways in his role of experiment representative. The SMS investigation was funded by NASA through contract NAS5-30370, by the Swiss National Science Foundation, and by the Bundesminister für Forschung und Technologie in Germany.

\section{References}

Balsiger, H., Eberhardt, P., Geiss, J., and Young, D. T.: 1980, J. Geophys. Res. 85, 1645.

Bonifazo, C. and Moreno, G.: 1981, J. Geophys. Res. 86, 4405.

Breneman, H. H. and Stone, E. C.: 1985, Astrophys. J. 299, L57.

Bürgi, A. and Geiss, J.: 1986, Solar Phys. 103, 347.

Burlaga, L. F.: 1984, Space Sci. Rev. 39, 255.

Chappel, C. R., Moore, T. E., and Wait, J. H., Jr.: 1987, J. Geophys. Res. 92, 5896.

Christon, S. P., Gloeckler, G., Williams, D. J., Mukai, T., McEntire, R. W., Jacquey, C., Angelopoulos, V., Lui, A. T. Y., Kokubun, S., Yamamoto, T., and Fairfield, D. H.: 1994, 'Energetic Atomic and Molecular Ions of Ionospheric Origin Observed in Distant Magnetotail Flow-Reversal Events', Geophys. Res. Letters (in press).

Fisk, L. A.: 1978, Astrophys. J. 224, 1048.

Galvin, A. B., Ipavich, F. M., Gloeckler, G., Hovestadt, D., Bame, S. J., Klecker, B., Scholer, M., and Tsurutani, B. T.: 1987, J. Geophys. Res. 92, 12069.

Geiss, J., Bühler, F., Cerutti, H., Eberhardt, P., and Filleux, C.: 1972, 'Sec. 14 of the Apollo 16 Preliminary Science Report', NASA SP-315.

Geiss, J. and Bürgi, A.: 1986, Astron. Astrophys. 159, 1.

Gloeckler, G.: 1977, 'A Versatile Detector System to Measure the Charge States, Mass Compositions, and Energy Spectra of Interplanetary and Magnetospheric Ions', University of Maryland, Technical Report TR 77-043 (unpublished).

Gloeckler, G.: 1990, Rev. Sci. Instrum. 61, 3613.

Gloeckler, G. and Geiss, J.: 1989, in J. C. Wadding (eds.), 'The Abundances of Elements and Isotopes in the Solar Wind', Cosmic Abundances of Matter, AIP Conf. Proceedings 183, 49.

Gloeckler, G. and Hamilton, D. C.: 1987, Physic Script T18, 73.

Gloeckler, G. and Hsieh, K. C.: 1979, Nucl. Instrum. Meth. $165,537$.

Gloeckler, G., Geiss, J., Balsiger, H., Fisk, L. A., Gliem, F., Ipavich, F. M., Ogilvie, K. W., Stüdemann, W., and Wilken, B.: 1983, 'The Solar Wind Ion Composition Spectrometer', ESA Spec. Publ. SP-1050 77.

Gloeckler, G., Hovestadt, D., Ipavich, F. M., Scholar, M., Klecker, B., and Galvin, A. B.: 1986, Geophys. Res. Letters 13, 251.

Gloeckler, G., Ipavich, F. M., Hamilton, D. C., Wilken, B., and Kremser, G.: 1989, EOS Trans. 70, 424.

Gloeckler, G., Geiss, J., Balsiger, H., Bedini, P., Cain, J. C., Fisher, J., Fisk, L. A., Galvin, A. B., Gliem, F., Hamilton, D. C., Hollweg, J. V., Ipavich, F. M., Joos, R., Livi, S., Lundgren, R., 
McKenzie, J. F., Mall, U., Ogilvie, K. W., Ottens, F., Rieck, W., Tums, E. O., von Steiger, R., Weiss, W., and Wilken, B.: 1992, Astron. Astrophys. Suppl. Ser. 92, 267.

Gloeckler, G., Geiss, J., Balsiger, H., Fisk, L. A., Galvin, A. B., Ipavich, F. M., Ogilvie, K. W., von Steiger, R., and Wilken, B.: 1993, Science 261, 70.

Gloeckler, G., Geiss, J., Roelof, E. C., Fisk, L. A., Ipavich, F. M., Ogilvie, K. W., Lanzerotti, L. J., von Steiger, R., and Wilken, B.: 1994, J. Geophys. Res. 99, 17637.

Hamilton, D. C., Gloeckler, G., Ipavich, G. M., Lundgren, R. A., Sheldon, R. B., and Hovestadt, D.: 1990, Rev. Sci. Instrum. 61(10), 3104.

Hilchenbach, M., Hovestadt, D., Klecker, B., and Möbius, E.: 1991, in E. Marsch and G. Schwenn (eds.), 'Detection of Singly Ionized Energetic Lunar Pick-Up Ions Upstream of the Earth's Bow Shock', Proceedings Solar Wind Seven, Pergamon Press, p. 349.

Hilchenbach, M., Hovestadt, D., Klecker, B., and Möbius, E.: 1992, Adv. Space Res. 13, 321.

Hollweg, J. V.: 1978, Rev. Geophys. Space Phys. 16, 689.

Hundhausen, A., Gilbert, H., and Bame, S.: 1968, J. Geophys. Res. 73, 5485.

Ipavich, F. M., Lundgren, R. A., Lambird, B. A., and Gloeckler, G.: 1978, Nucl. Inst. and Meth. 154, 291.

Ipavich, F. M., Galvin, A. B., Gloeckler, G., Scholer, M., and Hovestadt, D.: 1981, J. Geophys. Res. 86, 4337.

Ipavich, F. M., Galvin, A. B., Gloeckler, G., Hovestadt, D., Klecker, B., and Scholer, M.: 1986, Science 232, 366.

Isenberg, P. A.: 1991, J. Geophys. Res. 96, 155.

Isenberg, P. A. and Hollweg, J. V.: 1983, J. Geophys. Res. 88, 3923.

Lanzerotti, L. J., Gold, R. E., Anderson, K. A., Armstrong, T. P., Lin, R. P., Krimigis, S. M., Pick, M., Roelof, E. C., Sarris, E. T., Simnett, G. M., and Frain, W. E.: 1992, Astron. Astrophys. Suppl. Ser. 92, 349.

Lennartson, W. and Shelley, E. G.: 1986, J. Geophys. Res. 91, 3061.

Ogilvie, K. W., Bochsler, P., Geiss, J., and Coplan, M. A.: 1980, J. Geophys. Res. 85, 6069.

Paschmann, G., Sckopke, N., Papamastorakis, I., Asbridge, J. R., Bame, S. J., and Gosling, J. T.: 1981, J. Geophys. Res. 86, 4355.

Reames, D. V., Richardson, I. G., and Barbier, L. M.: 1991, Astrophys. J. 382, L43.

Sarris, E. T., Krimigis, S. M., Bostrom, C. O., and Armstrong, T. P.: 1978, J. Geophys. Res. 83 , 4289.

Schmidt, W. K. H., Rosenbauer, H., Shelley, E. G., and Geiss, J.: 1980, Geophys. Res. Letters 7, 697.

Tan, L. C., Mason, G. M., Klecker, B., and Hovestadt, D.: 1989, Astrophys. J. 345, 572.

von Steiger, R. and Geiss, J.: 1989, Astron. Astrophys. 225, 222.

von Steiger, R., Christon, S. P., Gloeckler, G., and Ipavich, F. M.: 1992, Astrophys. J. 389, 791.

Vauclair, S. and Meyer, J.-P.: 1985, 'Diffusion in the Chromosphere, and the Composition of the Solar Corona and Energetic Particles', Proceedings 19th Int. Cosmic Ray Conf. 4, 233.

Williams, D. J., McEntire, R. W., Schlemm, C., II, Lui, A. T. Y., Gloeckler, G., Christon, S. P., and Gliem, F.: 1994, J. Geomagn. Geoelect. 46, 39. 